\title{
Chiral susceptibility in the Nambu-Jona-Lasinio model: A Wigner function approach
}

\author{
Arpan Das, ${ }^{1, *}$ Deepak Kumar@ ${ }^{1,2, \dagger}$ and Hiranmaya Mishra ${ }^{1, \$}$ \\ ${ }^{1}$ Theory Division, Physical Research Laboratory, Navrangpura, Ahmedabad 380 009, India \\ ${ }^{2}$ Indian Institute of Technology Gandhinagar, Gandhinagar 382 355, Gujarat, India
}

(Received 9 August 2019; published 26 November 2019)

\begin{abstract}
We estimate here chiral susceptibility at finite temperature within the framework of the Nambu-JonaLasinio model (NJL) using the Wigner function approach. We also estimate it in the presence of chiral chemical potential $\left(\mu_{5}\right)$ as well as a nonvanishing magnetic field $(B)$. We use a medium separation regularization scheme (MSS) in the precence of magnetic field to calculate the chiral condensate and corresponding susceptibility. It is observed that for a fixed value of chiral chemical potential $\left(\mu_{5}\right)$, transition temperature increases with the magnetic field. While for the fixed value of the magnetic field, transition temperature decreases with chiral chemical potential. For a strong magnetic field, we observe nondegeneracy in susceptibility for up and down type quarks.
\end{abstract}

DOI: 10.1103/PhysRevD.100.094030

\section{INTRODUCTION}

In recent years, extensive efforts have been made to create and understand strongly interacting matter in relativistic heavy ion collision experiments, e.g., at the relativistic heavy-ion collider and the large hadron collider. There are mounting evidences which indicate formation of deconfined quark gluon plasma (QGP) phase of QCD in the initial stages of these experiments as well as the formation of confined hadron phase in the subsequent evolution of QGP. Ground state of QCD exhibits two main nonperturbative features, color confinement and spontaneous breaking of chiral symmetry. The dynamical breaking of chiral symmetry is the manifestation of the quark-antiquark condensation in the QCD vacuum. Dynamical chiral symmetry breaking characterizes the nonperturbative nature of QCD vacuum at vanishing temperature and/or density. With increase in temperature and/or baryon density, the QCD vacuum undergoes a transition from a chiral symmetry broken phase to a chiral symmetric phase. This transition is characterized by the quark-antiquark scalar condensate, the order parameter of the chiral phase transition. Although for first order phase transition order parameter changes discontinuously across the transition point, for second

\footnotetext{
*arpan@prl.res.in

†deepakk@prl.res.in

"hm@prl.res.in
}

Published by the American Physical Society under the terms of the Creative Commons Attribution 4.0 International license. Further distribution of this work must maintain attribution to the author(s) and the published article's title, journal citation, and DOI. Funded by SCOAP ${ }^{3}$. order phase transition or for a crossover transition the variation of order parameter across the transition point is rather smooth. In these cases, the fluctuation of this order parameter and the associated susceptibilities are more relevant for the characterization of the thermodynamic properties of the system.

The characteristics of fluctuations and correlations are intimately connected to the phase transition dynamics, e.g., fluctuations of all length scales are relevant at QCD critical point where the first order quark-hadron phase transition line ends. The study of fluctuations and correlations are essential phenomenological tool for the experimental exploration of the QCD phase diagram. In the context of heavy-ion collisions by studying the net electric charge fluctuation, it has been demonstrated that net electric charges are suppressed in the QGP phase as compared to the hadronic phase $[1,2]$. It has also been pointed out that the correlation between baryon number and strangeness is stronger in the QGP phase as compared to the hadronic phase $[3,4]$. The quantity of interest here is the chiral susceptibility which measures the response of the chiral condensate to the variation of the current quark mass. Chiral susceptibility has been calculated using first principle lattice QCD (LQCD) simulations [5-10]. All these lattice results show a pronounced peak in the variation of chiral susceptibility with temperature at the transition temperature, which essentially characterizes the chiral transition. Apart from these LQCD studies which incorporate the nonperturbative effects of QCD vacuum, complementary approaches, e.g., NambuJona-Lasinio (NJL) model $[11,12]$, chiral perturbation theory [13], Dyson-Schwinger equation [14], hard thermal loop approximation [15], etc. have been considered to study the chiral susceptibility. 
An entirely new line of investigations has been initiated to understand the QCD phase diagram due to the possibility of generation of extremely large magnetic field in noncentral relativistic heavy ion collision experiments. In the early stages, the magnetic field in QGP can be very large, at least of the order of few $m_{\pi}^{2}$ [16-24]. While such fields rapidly decay in the vacuum, in a conducting medium they can be sustained for a longer time due to induced current [21-24]. Strong magnetic field can affect dynamical chiral symmetry breaking. It has been shown that external magnetic field acts as catalysis for chiral condensation; the value of chiral condensation or the constituent mass of quarks is larger than vanishing magnetic field case. It is important to mention that the effect of magnetic field on the order parameter is not unique to QCD medium. In fact, in condensed matter systems, e.g., superconductors magnetic field can play a significant role. A striking contrast of the effect of magnetic field on the chiral condensate contrary to superconductors is that the magnetic field helps to strengthen the chiral condensate. Naively one can understand this in the following way. Unlike the electrically charged superconducting condensate, chiral condensate is an electrically neutral spin zero condensate. Hence, for the chiral condensate, the magnetic moment of the fermion and the antifermion point in the same direction. Hence, in the presence of magnetic field, both magnetic moments can align themselves along the direction of the magnetic field without any frustration in the pair [25]. It has also been pointed out that in the presence of magnetic field dimensional reduction can play an essential role in the pairing of fermions [26].

Magnetic catalysis has been explored extensively in $(2+1)$ and $(3+1)$-dimensional models with local four fermion interactions [27-46], supersymmetric models [47], quark meson models $[48,49]$, chiral perturbation theory $[50,51]$, etc. Such a strong magnetic field can also introduce some exotic phenomenon, e.g., chiral magnetic effect (CME), chiral vortical effect (CVE), etc. in a chirally imbalanced medium [52]. Underlying physics of the chiral imbalance is the axial anomaly and topologically nontrivial vacuum of $\mathrm{QCD}$, which allows topological field configurations like instantons to exist. An asymmetry between the number of left- and right-handed quarks can be generated by these nontrivial topological field configurations due to Adler-Bell-Jackiw anomaly. Such an imbalance can lead to observable $P$ and $C P$ violating effects in heavy ion collisions. In the presence of magnetic field, chirally imbalance quark matter can give rise to chiral magnetic effect where a charge separation can be produced. Effects of a chiral imbalance on the QCD phase diagram can be studied within the framework of grand canonical ensemble by introducing a chiral chemical potential $\mu_{5}$, which enters the QCD Lagrangian via a term $\mu_{5} \bar{\psi} \gamma^{0} \gamma^{5} \psi$. Chiral phase transition has been discussed extensively. These studies include NJL type models [53-59], quark linear sigma model [53,60], lattice QCD studies [61,62], etc. Although the effect of chiral chemical potential has been explored extensively, contradicting results have been reported in various literature, e.g., Refs. [53-58] predict that chiral transition temperature decreases with chiral chemical potential. On the other hand, in Ref. [59], it has been argued that with a specific regularization method chiral transition temperature increases with chiral chemical potential, which is in agreement with lattice results in Refs. [61,62]. In this context, in a recent interesting work, the Winger function in the presence of nonvanishing magnetic field and chiral chemical potential has been evaluated in a nonperturbative manner using explicit solutions of the Dirac equation in a magnetic field and chiral chemical potential [63]. This has been later used for pair production in the presence of electromagnetic field [64].

To probe the medium produced in relativistic heavy ion collisions, generally thermodynamic or hydrodynamic model has been used, which assumes local thermal equilibrium. However, due to the short timescales associated with the strong interaction, the medium produced in the heavy ion collision is rather dynamical in nature and lives for a very short time and nonequilibrium as well as quantum effects can affect the evolution of the medium significantly. These effects can be considered within the framework of nonequilibrium quantum transport theory. It is important to point out that in the case of interacting field theory of fermions and gauge bosons, transport theory should be invariant under local gauge transformation. Such a gauge covariant quantum transport theory for QCD has been developed in [65-67]. Classical kinetic theory is characterized by an ensemble of pointlike particles with their single particle phase-space distribution function. The time evolution of single particle phase-space distribution function governed by the transport equation encodes the evolution of the system. Similar to the single particle distribution in classical kinetic theory, Wigner function, which is the quantum mechanical analogue of classical distribution function, encodes quantum corrections in the transport equation [68]. Equation of motion of Winger function can be derived from the equation of motion for the associated field operators, e.g., for fermions, evolution equation of Wigner functions can be derived using the Dirac equation $[69,70]$. In the case of local gauge theories, the Wigner function has to be defined in a gauge invariant manner [71]. The covariant Wigner function method for spin- $1 / 2$ fermions has already been explored extensively in the context of heavy ion collisions to study various effects including the CME, CVE, polarization-vorticity coupling, hydrodynamics with spin, dynamical generation of magnetic moment, etc. [63,72-83].

In this investigation, we study the chiral phase transition and chiral susceptibility in the presence of magnetic field and chiral chemical potential in quantum kinetic theory framework using NJL model [84-89]. Our work is based on 
the spinor decomposition of the Wigner function using formalism of Refs. [63,90]. In this investigation, we limit ourselves to mean field or classical level of the quantum kinetic theory, since the chiral symmetry breaking and generation of dynamical mass of fermions take place at mean field level [90]. The formulation of transport theory of NJL model has been studied in Refs. [90-93]. In this work, we have used the formalism given in Ref. [90] to calculate the chiral condensate and the chiral susceptibility using the Wigner function. Wigner function in general is used for deriving dynamical equations for the out of equilibrium system [90]. In the present study, we limit ourselves to use the Wigner function for an extended system in global thermal equilibrium, i.e., at constant temperature and chemical potentials to calculate chiral susceptibility.

In this context, some comments regarding chiral transition in the presence of a of chiral chemical potential $\left(\mu_{5}\right)$ may be in order. In Ref. [54], this was investigated within Polyakov loop extended NJL (PNJL) model. It was observed that the chiral transition temperature decreases with chiral chemical potential. To eliminate artifacts of a sharp three momentum cutoff, in Ref. [54] a smooth cutoff for the three momentum modeled through a form factor was used. Further, it was observed that with increasing $\mu_{5}$ the chiral transition becomes a first order transition. In fact, the phase diagram in $\mu_{5}-T$ plane for the chiral transition becomes similar to the same in $\mu-T$ plane. This was also the conclusion in Refs. $[54,60,94]$. On the contrary, nonlocal version of the NJL model was further analyzed in Ref. [95] with the result that the chiral transition temperature increases with chiral chemical potential and the chiral transition is second order. Similar conclusion was also drawn in Refs. [96,97] using a Schwinger Dyson approach. Further, NJL model with chiral chemical potential was analyzed in Ref. [59] with a novel "medium separation scheme" (MSS) for regulating divergent integrals, and the conclusion was that the chiral transition temperature increases with $\mu_{5}$ and such conclusions are also in accordance with some lattice calculations $[61,62]$. However, it ought to be mentioned here that the lattice data have not been obtained in the chiral limit and some of the results are for $N_{c}=2$ QCD, e.g., [62]. A further careful analysis of NJL model was done in Ref. [57] to examine dependence of chiral transition temperature on different regularization scheme. It was observed that chiral transition temperature decreases with chiral chemical potential with a smooth cutoff and shows a first order transition at large $\mu_{5}$. In the present investigation, we use a medium separation scheme in the presence of magnetic field and chiral chemical potential. Such a scheme was introduced in Refs. $[59,98,99]$. As we will see later, we also do not see a first order transition at large chiral chemical potential as in the analysis in Ref. [95]. However, we observe that chiral transition temperature decreases with chiral chemical potential as in Refs. [54,57].
We organize the paper in the following manner. In Sec. II, for the sake of completeness, we recapitulate the results of Ref. [90] to study chiral condensate in NJL model using Wigner function approach. Then in Sec. III we introduce the Winger function in the presence of magnetic field as well as chiral chemical potential and calculate the chiral condensate for two flavor NJL model. In Sec. IV, we discuss the chiral susceptibility for two flavor NJL model in the presence of magnetic field $(B)$ as well as chiral chemical potential $\left(\mu_{5}\right)$. In Sec. $\mathrm{V}$, we present the results and discussions. Finally, in Sec. VI, we conclude our results with an outlook on it.

\section{WARM UP: WIGNER FUNCTION AND CHIRAL CONDENSATE IN NJL MODEL}

In this section we first briefly discuss the salient features of the formalism of Wigner function in NJL model for single flavor fermion having vanishing current quark mass as given in Ref. [90]. Once we get the representation of scalar condensate in terms of Wigner function, we can generalize it to a more realistic situation with nonvanishing current quark mass. For a single flavor NJL model, we start with the following Lagrangian [90]:

$$
\mathcal{L}=\bar{\psi} i \not \partial \psi+G\left((\bar{\psi} \psi)^{2}+\left(\bar{\psi} i \gamma_{5} \psi\right)^{2}\right)
$$

where $\psi$ is the Dirac field, $G$ is the scalar coupling. The first term is the usual kinetic term, and the second term represents the four Fermi interaction. One can define composite field operators $\hat{\sigma}$ and $\hat{\pi}$ as

$$
\hat{\sigma}=-2 G \bar{\psi} \psi, \quad \hat{\pi}=-2 G \bar{\psi} i \gamma_{5} \psi .
$$

Using Eq. (2), the Lagrangian given in Eq. (1) can be recasted as [90]

$$
\mathcal{L}=\bar{\psi} i \not \partial \psi-\hat{\sigma} \bar{\psi} \psi-\hat{\pi} \bar{\psi} i \gamma_{5} \psi-\frac{\hat{\sigma}^{2}+\hat{\pi}^{2}}{4 G} .
$$

In the mean field approximation, the operators $\hat{\sigma}$ and $\hat{\pi}$ are replaced by their mean field values

$\hat{\sigma} \rightarrow \sigma=\langle\hat{\sigma}\rangle=\operatorname{Tr}(\hat{\rho} \hat{\sigma}), \quad \hat{\pi} \rightarrow \pi=\langle\hat{\pi}\rangle=\operatorname{Tr}(\hat{\rho} \hat{\pi})$,

where $\hat{\rho}$ is the density matrix operator and " $\operatorname{Tr}$ " denotes trace over all physical states of the system. For a nonequilibrium transport theory, in mean field approximation, the fundamental quantity is the Green function, which is defined as

$$
G_{\alpha \beta}^{<}(x, y)=\left\langle\bar{\psi}_{\beta}(y) \psi_{\alpha}(x)\right\rangle .
$$


The mean field values of the operators $\hat{\sigma}$ and $\hat{\pi}$, i.e., $\sigma(x)$ and $\pi(x)$ can be determined in terms of the Green function $G^{<}(x, y)$ as follows:

$\sigma(x)=-2 G \operatorname{Tr} G^{<}(x, x), \quad \pi(x)=-2 G \operatorname{Tr} i \gamma_{5} G^{<}(x, x)$.

The Wigner function for fermion is defined as [90]

$$
\begin{aligned}
W_{\alpha \beta}(X, p) & =\int \frac{d^{4} X^{\prime}}{(2 \pi)^{4}} e^{-i p_{\mu} X^{\prime \mu}}\left\langle\bar{\psi}_{\beta}\left(X+\frac{X^{\prime}}{2}\right) \psi_{\alpha}\left(X-\frac{X^{\prime}}{2}\right)\right\rangle \\
& =\int \frac{d^{4} X^{\prime}}{(2 \pi)^{4}} e^{-i p_{\mu} X^{\prime \mu}} G_{\alpha \beta}^{<}\left(X+\frac{X^{\prime}}{2}, X-\frac{X^{\prime}}{2}\right)
\end{aligned}
$$

It is important to mention that in NJL model there are no gluons; hence, the $S U(3)_{c}$ gauge invariance of the Wigner function does not appear in NJL model. Again, in this case, we are not considering background magnetic field. Hence, there is no $U(1)_{e m}$ gauge field associated with the NJL model. However, in the presence of gauge field, one has to introduce a gauge link in Wigner function for a gauge invariant description [100].

Since the Wigner function $(W(X, p))$, as given in Eq. (7), is a composite operator made out of the Dirac field operators $\psi$ and $\bar{\psi}$, it is convenient to decompose $W(X, p)$ in terms of the generators of the Clifford algebra. The Wigner function $W(X, p)$, in terms of the conventional basis of Clifford algebra $\mathbb{1}, i \gamma_{5}, \gamma^{\mu}, \gamma^{\mu} \gamma_{5}$, and $\sigma^{\mu \nu}$, can be written as

$$
W=\frac{1}{4}\left[F+i \gamma_{5} P+\gamma^{\mu} V_{\mu}+\gamma^{\mu} \gamma^{5} A_{\mu}+\frac{1}{2} \sigma^{\mu \nu} S_{\mu \nu}\right] .
$$

Here the coefficients $F, P, V_{\mu}, A_{\mu}$, and $S_{\mu \nu}$ are the scalar, pseudoscalar, vector, axial vector, and tensor components of the Wigner function, respectively, also known as Dirac-Heisenberg-Wigner (DHW) functions. The scalar, pseudoscalar, vector, axial vector, and tensor Dirac-Heisenberg-Wigner functions can be, respectively, expressed as

$$
\begin{gathered}
F(X, p)=\operatorname{Tr} W(X, p), \\
P(X, p)=-i \operatorname{Tr} \gamma_{5} W(X, p), \\
V^{\mu}(X, p)=\operatorname{Tr} \gamma^{\mu} W(X, p), \\
A^{\mu}(X, p)=\operatorname{Tr} \gamma^{5} \gamma^{\mu} W(X, p), \\
S^{\mu \nu}(X, p)=\operatorname{Tr} \sigma^{\mu \nu} W(X, p) .
\end{gathered}
$$

Using Eqs. (6) and (7), the scalar and pseudoscalar condensates as given in Eqs. (9) and (10) can be written in terms of Wigner function in the following manner:

$\sigma(X)=-2 G \int d^{4} p \operatorname{Tr} W(X, p)=-2 G \int d^{4} p F(X, p)$,

and

$$
\pi(X)=-2 G \int d^{4} p \operatorname{Tr} i \gamma_{5} W(X, p)=2 G \int d^{4} p P(X, p) .
$$

Using Eqs. (2) and (14), one can express the scalar condensate as

$$
\langle\bar{\psi} \psi\rangle=\int d^{4} p F(X, p) .
$$

In the above description, we have briefly mentioned the relation between the different mean fields with the Wigner function. It is important to mention that by the virtue of the Dirac equation for the field operator $\psi$ and $\bar{\psi}$ the Wigner function, $W(X, p)$, also satisfies a quantum kinetic equation. However, in this investigation, we have not focused on the kinetic equation of the Wigner function. For a detailed discussion on the kinetic equation for the components of Wigner function, kinetic equation for quark distribution function, and related topic, see Ref. [90]. In this investigation, we rather focus on the estimation of chiral condensate, as given in Eq. (16), and associated chiral susceptibility in two flavor NJL model.

The Wigner function can be calculated by inserting the Dirac field operators in Eq. (7). The Dirac field operators in the absence of magnetic field can be written as [101]

$$
\begin{aligned}
\psi(x)= & \frac{1}{\sqrt{\Omega}} \sum_{\vec{k}, s} \frac{1}{\sqrt{2 \mathcal{E}_{0 k}}}\left[a(\vec{k}, s) u(\vec{k}, s) e^{-i k \cdot x}\right. \\
& \left.+b^{\dagger}(\vec{k}, s) v(\vec{k}, s) e^{i k \cdot x}\right], \\
\bar{\psi}(x)= & \frac{1}{\sqrt{\Omega}} \sum_{\vec{k}, s} \frac{1}{\sqrt{2 \mathcal{E}_{0 k}}}\left[a^{\dagger}(\vec{k}, s) \bar{u}(\vec{k}, s) e^{i k \cdot x}\right. \\
& \left.+b(\vec{k}, s) \bar{v}(\vec{k}, s) e^{-i k \cdot x}\right],
\end{aligned}
$$

where $\Omega$ is the volume and $s= \pm 1$ denotes the spin states. Using the field decomposition as given in Eqs. (17) and (18), the Wigner function of a fermion with mass $\mathcal{M}_{0}$ can be shown to be [101] 


$$
\begin{aligned}
W_{\alpha \beta}(X, p)= & \frac{1}{(2 \pi)^{3}} \delta\left(p^{2}-\mathcal{M}_{0}^{2}\right)\left[\theta\left(p^{0}\right) \sum_{s} f_{F D}\left(\mathcal{E}_{0 p}-\mu_{s}\right) u_{\alpha}(\vec{p}, s) \bar{u}_{\beta}(\vec{p}, s)\right. \\
& \left.+\theta\left(-p^{0}\right) \sum_{s}\left(1-f_{F D}\left(\mathcal{E}_{0 p}+\mu_{s}\right)\right) v_{\alpha}(-\vec{p}, s) \bar{v}_{\beta}(-\vec{p}, s)\right]
\end{aligned}
$$

where the creation and the annihilation operators of the particle satisfy $\left\langle a^{\dagger}(\vec{p}, s) a(\vec{p}, s)\right\rangle=f_{F D}\left(\mathcal{E}_{0 p}-\mu_{s}\right)$. On the other hand, the creation and the annihilation operators of the antiparticle satisfy $\left\langle b^{\dagger}(-\vec{p}, s) b(-\vec{p}, s)\right\rangle=f_{F D}\left(\mathcal{E}_{0 p}+\mu_{s}\right)$. Here $f_{F D}(z)=1 /(1+\exp (z / T))$ is the Fermi Dirac distribution function at temperature $T$, and $\mu_{s}$ is the chemical potential for the spin state $s . \mathcal{E}_{0 p}=\sqrt{p^{2}+\mathcal{M}_{0}^{2}}$ is the single particle energy, and $\mathcal{M}_{0}$ is the mass of the Dirac fermion. It is important to note that the space time dependence in the
Wigner function $W(X, p)$ is hidden in the space time dependence of the temperature and chemical potential. However, for a uniform temperature and chemical potential, i.e., for a system in global equilibrium the Wigner function is independent of space time. In this investigation, we considered a global thermal equilibrium. Hence, from now onward, we will omit the space time dependence in the Wigner function. Using Eqs. (9) and (19), the scalar DHW function can be expressed as [101]

$$
F(p)=\mathcal{M}_{0} \delta\left(p^{2}-\mathcal{M}_{0}^{2}\right)\left[\frac{2}{(2 \pi)^{3}} \sum_{s}\left(\theta\left(p^{0}\right) f_{F D}\left(\mathcal{E}_{0 p}-\mu_{s}\right)-\theta\left(-p^{0}\right)\left(1-f_{F D}\left(\mathcal{E}_{0 p}+\mu_{s}\right)\right)\right)\right]
$$

Using the scalar DHW function as given in Eq. (20), the scalar condensate for a single fermion species of mass $\mathcal{M}_{0}$ given in Eq. (16) can be recasted as

$$
\begin{aligned}
\langle\bar{\psi} \psi\rangle & =\int d^{4} p \mathcal{M}_{0} \delta\left(p^{2}-\mathcal{M}_{0}^{2}\right)\left[\frac{2}{(2 \pi)^{3}} \sum_{s}\left(\theta\left(p^{0}\right) f_{F D}\left(\mathcal{E}_{0 p}-\mu_{s}\right)-\theta\left(-p^{0}\right)\left(1-f_{F D}\left(\mathcal{E}_{0 p}+\mu_{s}\right)\right)\right)\right] \\
& =-\sum_{s} \int \frac{d^{3} p}{(2 \pi)^{3}} \frac{\mathcal{M}_{0}}{\mathcal{E}_{0 p}}\left[1-f_{F D}\left(\mathcal{E}_{0 p}-\mu_{s}\right)-f_{F D}\left(\mathcal{E}_{0 p}+\mu_{s}\right)\right] .
\end{aligned}
$$

In a situation where the chemical potential is independent of the spin of the state,

$$
\langle\bar{\psi} \psi\rangle=-2 N_{c} \int \frac{d^{3} p}{(2 \pi)^{3}} \frac{\mathcal{M}_{0}}{\mathcal{E}_{0 p}}\left[1-f_{F D}\left(\mathcal{E}_{0 p}-\mu\right)-f_{F D}\left(\mathcal{E}_{0 p}+\mu\right)\right], \quad \text { with } \quad \mathcal{M}_{0}=-2 G\langle\bar{\psi} \psi\rangle .
$$

The factor of $N_{c}$ appears in Eq. (22) due to the "Tr" over all the degrees of freedom (d.o.f.).

Next, we shall consider two flavor $(u, d$ quarks) NJL model for vanishing magnetic field and chiral chemical potential, with the Lagrangian given as [102-104], along with a 't Hooft determinant interaction

$$
\mathcal{L}=\mathcal{L}_{0}+\mathcal{L}_{1}+\mathcal{L}_{2}
$$

where the free part is

$$
\mathcal{L}_{0}=\bar{\psi}(i \not \supset-m) \psi
$$

and the interaction parts are given as

$$
\mathcal{L}_{1}=G_{1} \sum_{a=0}^{3}\left[\left(\bar{\psi} \tau^{a} \psi\right)^{2}+\left(\bar{\psi} i \gamma_{5} \tau^{a} \psi\right)^{2}\right]
$$

and

$\mathcal{L}_{2}=G_{2}\left[(\bar{\psi} \psi)^{2}-(\bar{\psi} \vec{\tau} \psi)^{2}-\left(\bar{\psi} i \gamma_{5} \psi\right)^{2}+\left(\bar{\psi} i \gamma_{5} \vec{\tau} \psi\right)^{2}\right]$,

where $\psi=\left(\psi_{u}, \psi_{d}\right)^{T}$ is the quark doublet, $m=$ $\operatorname{diag}\left(m_{u}, m_{d}\right)$ is the current quark mass with $m_{u}=m_{d}$. $\tau^{0}=I_{2 \times 2}$ and $\vec{\tau}$ are the Pauli matrices. The above Lagrangian as given in Eq. (23) is invariant under $S U(2)_{L} \times S U(2)_{R} \times U(1)_{V}$ transformations. $\mathcal{L}_{1}$ has an additional $U(1)_{A}$ symmetry. $\mathcal{L}_{2}$ is identical with 't Hooft determinant interaction term which breaks the $U(1)_{A}$ symmetry explicitly. $\mathcal{L}_{2}$ interaction term introduces mixing between different flavors. It is also important to emphasize that since we are considering only the scalar condensates of the form $\left\langle\bar{\psi}_{u} \psi_{u}\right\rangle$ and $\left\langle\bar{\psi}_{d} \psi_{d}\right\rangle$, so we can safely ignore the pseudoscalar condensate as well as the scalar condensates 
of the form $\left\langle\bar{\psi}_{u} \psi_{d}\right\rangle,\left\langle\bar{\psi}_{d} \psi_{u}\right\rangle$ etc. Using these approximations at the mean field level, the Lagrangian of the two flavor NJL model as given in Eq. (23) can be expressed as

$$
\begin{aligned}
\mathcal{L}= & \bar{\psi}_{u}\left(i \not \partial-\mathcal{M}_{0_{u}}\right) \psi_{u}+\bar{\psi}_{d}\left(i \not \partial-\mathcal{M}_{0_{d}}\right) \psi_{d} \\
& -2 G_{1}\left(\left\langle\bar{\psi}_{u} \psi_{u}\right\rangle^{2}+\left\langle\bar{\psi}_{d} \psi_{d}\right\rangle^{2}\right)-4 G_{2}\left\langle\bar{\psi}_{u} \psi_{u}\right\rangle\left\langle\bar{\psi}_{d} \psi_{d}\right\rangle,
\end{aligned}
$$

where $u$ and $d$ quark condensates are given as $\left\langle\bar{\psi}_{u} \psi_{u}\right\rangle$ and $\left\langle\bar{\psi}_{d} \psi_{d}\right\rangle$, respectively. The constituent quark masses of $u$ and $d$ quarks in terms of the chiral condensates are given as

$$
\begin{aligned}
& \mathcal{M}_{0_{u}}=m_{u}-4 G_{1}\left\langle\bar{\psi}_{u} \psi_{u}\right\rangle-4 G_{2}\left\langle\bar{\psi}_{d} \psi_{d}\right\rangle, \\
& \mathcal{M}_{0_{d}}=m_{d}-4 G_{1}\left\langle\bar{\psi}_{d} \psi_{d}\right\rangle-4 G_{2}\left\langle\bar{\psi}_{u} \psi_{u}\right\rangle .
\end{aligned}
$$

One can easily generalize the scalar condensate as given in Eq. (22) for single flavor NJL model to multiflavor NJL model. Hence, for NJL model of $N_{f}$ quark flavor and $N_{c}$ color, the chiral condensate can be written as

$$
\langle\bar{\psi} \psi\rangle_{B=0}^{\mu_{5}=0}=\sum_{f=1}^{N_{f}}\left\langle\bar{\psi}_{f} \psi_{f}\right\rangle_{B=0}^{\mu_{5}=0},
$$

with

$$
\begin{aligned}
\left\langle\overline{\psi_{f}} \psi_{f}\right\rangle_{B=0}^{\mu_{5}=0}= & -2 N_{c} \int \frac{d^{3} p}{(2 \pi)^{3}} \frac{\mathcal{M}_{0_{f}}}{\mathcal{E}_{0 p, f}}\left[1-f_{F D}\left(\mathcal{E}_{0 p, f}-\mu\right)\right. \\
& \left.-f_{F D}\left(\mathcal{E}_{0 p, f}+\mu\right)\right] .
\end{aligned}
$$

The chiral condensate for $N_{f}$ flavor NJL model as given in Eq. (29) can also be obtained by first calculating the thermodynamic potential using the mean field Lagrangian as given in Eq. (27) and then calculating the gap equation using the minimization of thermodynamic potential.

\section{WIGNER FUNCTION AND CHIRAL CONDENSATE IN NJL MODEL FOR NONVANISHING MAGNETIC FIELD AND CHIRAL CHEMICAL POTENTIAL}

In the presence of magnetic field $(B)$ and chiral chemical potential $\left(\mu_{5}\right)$, the Wigner function has been explicitly written down in Ref. [63], using solutions of the Dirac equation for fermions in magnetic field and finite chiral chemical potential. We shall use them to calculate chiral condensate. For the sake of completeness, we write down the relevant expressions for the Wigner function. In the presence of background magnetic field, the Wigner function given in Eq. (7) gets modified to a gauge invariant Wigner function as [63]

$$
\begin{aligned}
W_{\alpha \beta}(X, p)= & \int \frac{d^{4} X^{\prime}}{(2 \pi)^{4}} e^{\left(-i p_{\mu} X^{\prime \mu}\right)}\left\langle\bar{\psi}_{\beta}\left(X+\frac{X^{\prime}}{2}\right)\right. \\
& \left.\times U\left(A, X+\frac{X^{\prime}}{2}, X-\frac{X^{\prime}}{2}\right) \psi_{\alpha}\left(X-\frac{X^{\prime}}{2}\right)\right\rangle,
\end{aligned}
$$

where $U\left(A, X+\frac{X^{\prime}}{2}, X-\frac{X^{\prime}}{2}\right)$ is the gauge link between two space time points $\left(X-\frac{X^{\prime}}{2}\right)$ and $\left(X+\frac{X^{\prime}}{2}\right)$ for the gauge field $A^{\mu}$. The gauge link has been introduced to make the Wigner function gauge invariant. In the presence of homogeneous external magnetic field along the $z$ direction, the gauge link is just a phase. In this case, the Wigner function simplifies to

$$
\begin{aligned}
W_{\alpha \beta}(X, p)= & \int \frac{d^{4} X^{\prime}}{(2 \pi)^{4}} e^{\left(-i p_{\mu} X^{\prime \mu}-i q B y x^{\prime}\right)}\left\langle\bar{\psi}_{\beta}\left(X+\frac{X^{\prime}}{2}\right)\right. \\
& \left.\otimes \psi_{\alpha}\left(X-\frac{X^{\prime}}{2}\right)\right\rangle,
\end{aligned}
$$

where $A^{\mu}(X)=(0,-B y, 0,0)$ is a specific gauge choice of the external magnetic field. $q$ is the charge of the particle, and it has been taken to be positive. Analogous to the case of vanishing magnetic field, Wigner function can be calculated for nonvanishing magnetic field by using the Dirac field operator in a background magnetic field. The Wigner function in a background magnetic field at finite temperature $(T)$, chemical potential $(\mu)$, and finite chiral chemical potential $\left(\mu_{5}\right)$ has been shown to be [63]

$$
\begin{aligned}
W(p)= & \sum_{n, s}\left[f_{F D}\left(E_{p_{z}, s}^{(n)}-\mu\right) \delta\left(p_{0}+\mu-E_{p_{z}, s}^{(n)}\right) W_{+, s}^{(n)}(\vec{p})\right. \\
& \left.+\left(1-f_{F D}\left(E_{p_{z}, s}^{(n)}+\mu\right)\right) \delta\left(p_{0}+\mu+E_{p_{z}, s}^{(n)}\right) W_{-, s}^{(n)}(\vec{p})\right],
\end{aligned}
$$$$
n \geq 0 \text {, }
$$

where the functions $W_{ \pm, s}^{(n)}(\vec{p})$ denote the contribution of fermion/antifermion in the $n$th Landau level. The single particle energy at the lowest Landau level and higher Landau level is given as $E_{p_{z}}^{(0)}=\sqrt{M^{2}+\left(p_{z}-\mu_{5}\right)^{2}}$ and $E_{p_{z}, s}^{(n)}=\sqrt{M^{2}+\left(\sqrt{p_{z}^{2}+2 n q B}-s \mu_{5}\right)^{2}}$, respectively. + and - in Eq. (32) denote contributions of positive and negative energy solutions, respectively. In the lowest Landau level, fermions can only be in a specific spin state. On the other hand, for higher Landau levels $(n>0)$, both spin states contribute.

The functions $W_{ \pm, s}^{(n)}(\vec{p})$ in Eq. (32) can be expressed in terms of Dirac spinors in the following manner [63]:

$$
\begin{aligned}
W_{r s}^{(n)}(\vec{p}) \equiv & \frac{1}{(2 \pi)^{3}} \int d y^{\prime} \exp \left(i p_{y} y^{\prime}\right) \xi_{r s}^{(n) \dagger}\left(p_{x}, p_{z}, \frac{y^{\prime}}{2}\right) \gamma^{0} \\
& \otimes \xi_{r s}^{(n)}\left(p_{x}, p_{z},-\frac{y^{\prime}}{2}\right), \quad n \geq 0 .
\end{aligned}
$$


In Eq. (33), $r= \pm$ denotes positive energy and negative energy solutions, respectively. The Dirac spinors $\xi_{r}^{(0)}$ and $\xi_{r s}^{(n)}$, where $r= \pm$ denotes positive and negative energy states and $s$ denotes the spin of the state, are defined as

$$
\begin{gathered}
\xi_{r}^{(0)}\left(p_{x}, p_{z}, y\right)=\frac{1}{\sqrt{2 E_{p_{z}}^{(0)}}}\left(\begin{array}{c}
r \sqrt{E_{p_{z}}^{(0)}-r\left(p_{z}-\mu_{5}\right)} \\
\sqrt{E_{p_{z}}^{(0)}+r\left(p_{z}-\mu_{5}\right)}
\end{array}\right) \otimes \chi^{(0)}\left(p_{x}, y\right), \\
\xi_{r s}^{(n)}\left(p_{x}, p_{z}, y\right)=\frac{1}{\sqrt{2 E_{p_{z}, s}^{(n)}}}\left(\begin{array}{l}
r \sqrt{E_{p_{z}, s}^{(n)}+r \mu_{5}-r s \sqrt{p_{z}^{2}+2 n q B}} \\
\sqrt{E_{p_{z}, s}^{(n)}-r \mu_{5}+r s \sqrt{p_{z}^{2}+2 n q B}}
\end{array}\right) \otimes \chi^{(n)}\left(p_{x}, p_{z}, y\right), \quad n>0,
\end{gathered}
$$

where the normalized eigen spinors $\chi$ are

$$
\chi^{(0)}\left(p_{x}, y\right)=\left(\begin{array}{l}
1 \\
0
\end{array}\right) \phi_{0}\left(p_{x}, y\right)
$$

and

$$
\chi_{s}^{(n)}\left(p_{x}, p_{z}, y\right)=\frac{1}{\sqrt{2 \sqrt{p_{z}^{2}+2 n q B}}}\left(\begin{array}{c}
\sqrt{\sqrt{p_{z}^{2}+2 n q B}+s p_{z}} \phi_{n}\left(p_{x}, y\right) \\
s \sqrt{\sqrt{p_{z}^{2}+2 n q B}-s p_{z}} \phi_{n-1}\left(p_{x}, y\right)
\end{array}\right), \quad n>0
$$

where

$$
\phi_{n}\left(p_{x}, y\right)=\left(\frac{q B}{\pi}\right)^{1 / 4} \frac{1}{\sqrt{2^{n} n !}} \exp \left[-\frac{q B}{2}\left(y+\frac{p_{x}}{q B}\right)^{2}\right] H_{n}\left[\sqrt{q B}\left(y+\frac{p_{x}}{q B}\right)\right], \quad n \geq 0 .
$$

$H_{n}$ represents $n$th Hermite polynomial. Inserting the explicit expression of the Dirac spinors as given in Eqs. (36) and (37) into Eq. (33), one can get the explicit form of the function $W_{ \pm, s}^{(n)}(\vec{p})$ [63]. For lowest Landau level,

$$
W_{r}^{(0)}(\vec{p})=\frac{r}{4(2 \pi)^{3} E_{p_{z}}^{(0)}} \Lambda^{(0)}\left(p_{T}\right)\left[M\left(1+\sigma^{12}\right)+r E_{p_{z}}^{(0)}\left(\gamma^{0}-\gamma^{5} \gamma^{3}\right)-\left(p_{z}-\mu_{5}\right)\left(\gamma^{3}-\gamma^{5} \gamma^{0}\right)\right]
$$

while for higher Landau levels,

$$
\begin{aligned}
W_{r s}^{(n)}(\vec{p})= & r \frac{1}{4(2 \pi)^{3} E_{p_{z}, s}^{(n)}}\left\{\left[\Lambda_{+}^{(n)}\left(p_{T}\right)+s \frac{p_{z}}{\sqrt{p_{z}^{2}+2 n q B}} \Lambda_{-}^{(n)}\left(p_{T}\right)\right]\left[M+r E_{p_{z}, s}^{(n)} \gamma^{0}+\left(s \sqrt{p_{z}^{2}+2 n q B}-\mu_{5}\right) \gamma^{5} \gamma^{0}\right]\right. \\
& -\left[\Lambda_{-}^{(n)}\left(p_{T}\right)+s \frac{p_{z}}{\sqrt{p_{z}^{2}+2 n q B}} \Lambda_{+}^{(n)}\left(p_{T}\right)\right]\left[\left(s \sqrt{p_{z}^{2}+2 n q B}-\mu_{5}\right) \gamma^{3}+r E_{p_{z}, s}^{(n)} \gamma^{5} \gamma^{3}-M \sigma^{12}\right] \\
& -\frac{2 n q B}{p_{T}^{2} \sqrt{p_{z}^{2}+2 n q B}} \Lambda_{+}^{(n)}\left(p_{T}\right)\left[\left(\sqrt{p_{z}^{2}+2 n q B}-s \mu_{5}\right)\left(p_{x} \gamma^{1}+p_{y} \gamma^{2}\right)\right. \\
& \left.\left.+r s E_{p_{z}, s}^{(n)}\left(p_{x} \gamma^{5} \gamma^{1}+p_{y} \gamma^{5} \gamma^{2}\right)-s M\left(p_{z} \sigma^{23}-p_{y} \sigma^{13}\right)\right]\right\}, \quad n>0,
\end{aligned}
$$

where

$$
\Lambda_{ \pm}^{(0)}\left(p_{T}\right)=2 \exp \left(-\frac{p_{T}^{2}}{q B}\right)
$$




$$
\Lambda_{ \pm}^{(n)}\left(p_{T}\right)=(-1)^{n}\left[L_{n}\left(\frac{2 p_{T}^{2}}{q B}\right) \mp L_{n-1}\left(\frac{2 p_{T}^{2}}{q B}\right)\right] \exp \left(-\frac{p_{T}^{2}}{q B}\right), \quad n>0
$$

Here $L_{n}(x)$ are the Laguerre polynomials with $L_{-1}(x)=0$. Using the Wigner function $W(p)$, as given in Eq. (32), it can be shown that the scalar DWH function is [63]

$$
F(p)=M\left[\sum_{n=0}^{\infty} V_{n}\left(p_{0}, p_{z}\right) \Lambda_{+}^{(n)}\left(p_{T}\right)+\sum_{n=1}^{\infty} \frac{1}{\sqrt{p_{z}^{2}+2 n q B}} A_{n}\left(p_{0}, p_{z}\right) p_{z} \Lambda_{-}^{(n)}\left(p_{T}\right)\right]
$$

where

$$
\begin{gathered}
V_{0}\left(p_{0}, p_{z}\right)=\frac{2}{(2 \pi)^{3}} \delta\left\{\left(p_{0}+\mu\right)^{2}-\left|E_{p_{z}}^{(0)}\right|^{2}\right\}\left\{\theta\left(p_{0}+\mu\right) f_{F D}\left(p_{0}\right)+\theta\left(-p_{0}-\mu\right)\left[f_{F D}\left(-p_{0}\right)-1\right]\right\} \\
V_{n}\left(p_{0}, p_{z}\right)=\frac{2}{(2 \pi)^{3}} \sum_{s} \delta\left\{\left(p_{0}+\mu\right)^{2}-\left|E_{p_{z}, s}^{(n)}\right|^{2}\right\}\left\{\theta\left(p_{0}+\mu\right) f_{F D}\left(p_{0}\right)+\theta\left(-p_{0}-\mu\right)\left[f_{F D}\left(-p_{0}\right)-1\right]\right\}, \quad n>0 \\
A_{n}\left(p_{0}, p_{z}\right)=\frac{2}{(2 \pi)^{3}} \sum_{s} s \delta\left\{\left(p_{0}+\mu\right)^{2}-\left|E_{p_{z}, s}^{(n)}\right|^{2}\right\}\left\{\theta\left(p_{0}+\mu\right) f_{F D}\left(p_{0}\right)+\theta\left(-p_{0}-\mu\right)\left[f_{F D}\left(-p_{0}\right)-1\right]\right\}, \quad n>0 .
\end{gathered}
$$

Once the scalar DWH function is known explicitly as given in Eq. (43), the chiral condensate of single flavor fermion can be calculated using Eq. (16) and is given as

$$
\langle\bar{\psi} \psi\rangle=\int d^{4} p F(p)=\int 2 \pi p_{T} d p_{0} d p_{T} d p_{z} F(p) .
$$

Using Eqs. (43) and (47), it can be shown that (see Appendix A for details)

$$
\begin{aligned}
\langle\bar{\psi} \psi\rangle_{B \neq 0}^{\mu_{5} \neq 0}= & -\frac{q B}{(2 \pi)^{2}}\left[\int d p_{z} \frac{M}{E_{p_{z}}^{(0)}}\left[1-f_{F D}\left(E_{p_{z}}^{(0)}-\mu\right)-f_{F D}\left(E_{p_{z}}^{(0)}+\mu\right)\right]\right. \\
& \left.+\sum_{n=1}^{\infty} \sum_{s} \int d p_{z} \frac{M}{E_{p_{z}, s}^{(n)}}\left[1-f_{F D}\left(E_{p_{z}, s}^{(n)}-\mu\right)-f_{F D}\left(E_{p_{z}, s}^{(n)}+\mu\right)\right]\right] .
\end{aligned}
$$

For vanishing chiral chemical potential, $\mu_{5}=0$, scalar condensate gets reduced to

$$
\langle\bar{\psi} \psi\rangle_{B \neq 0}^{\mu_{5}=0}=-\frac{q B}{(2 \pi)^{2}} \sum_{n=0}^{\infty}\left(2-\delta_{n, 0}\right) \int d p_{z} \frac{M_{0}}{\epsilon_{p_{z}}^{(n)}}\left[1-f_{F D}\left(\epsilon_{p_{z}}^{(n)}-\mu\right)-f_{F D}\left(\epsilon_{p_{z}}^{(n)}+\mu\right)\right],
$$

where we denote $M_{0}$ as the mass of fermion in the absence of chiral chemical potential and finite magnitude field. The single particle energy $\epsilon_{p_{z}}^{(n)}$ for vanishing chiral chemical potential can be written as

$$
\epsilon_{p_{z}}^{(n)}=\sqrt{M_{0}^{2}+p_{z}^{2}+2 n q B}, \quad n \geq 0
$$

The chiral condensate for a single flavor as given in Eq. (48) can be easily extended to NJL model with two flavors. Most general Lagrangian for two flavor NJL model with $u$ and $d$ quarks in the magnetic field including chiral chemical potential is given as

$\mathcal{L}=\bar{\psi}\left(i \not D-m+\mu_{5} \gamma^{0} \gamma^{5}\right) \psi+G_{1} \sum_{a=0}^{3}\left[\left(\bar{\psi} \tau^{a} \psi\right)^{2}+\left(\bar{\psi} i \gamma_{5} \tau^{a} \psi\right)^{2}\right]+G_{2}\left[(\bar{\psi} \psi)^{2}-(\bar{\psi} \vec{\tau} \psi)^{2}-\left(\bar{\psi} i \gamma_{5} \psi\right)^{2}+\left(\bar{\psi} i \gamma_{5} \vec{\tau} \psi\right)^{2}\right]$

where $\psi$ is the $U(2)$ quark doublet, given as $\psi=\left(\psi_{u}, \psi_{d}\right)^{T}$. The covariant derivative is given as $\not D=\not \supset+i q \not A$, and the current quark mass matrix is $m=\operatorname{diag}\left(m_{u}, m_{d}\right)$, with $m_{u}=m_{d}$. The first term in Eq. (51) is the free Dirac Lagrangian in the 
presence of magnetic field. For the calculation, we have considered the gauge choice of the background magnetic field as $A^{\mu}=(0,-B y, 0,0)$. The second term in Eq. (51) is the four Fermi interaction and the attractive part of the quark-antiquark channel of the Fierz transformed color current-current interaction. $\tau^{a}, a=0, \ldots 3$ are the $U(2)$ generators in the flavor space. The third term is the 't Hooft interaction term which introduces flavor mixing, as in Eq. (26). Since the magnetic field couples to the electric charge of particles, in the presence of magnetic field, $u$ quark and $d$ quarks couple differently with the magnetic field; hence, the isospin symmetry is explicitly broken. In the mean field approximation, in the absence of any pseudoscalar condensate, Eq. (51) can be recasted as

$$
\begin{aligned}
\mathcal{L}= & \bar{\psi}_{u}\left(i \not D-M_{u}+\mu_{5} \gamma^{0} \gamma^{5}\right) \psi_{u}+\bar{\psi}_{d}\left(i \not D-M_{d}+\mu_{5} \gamma^{0} \gamma^{5}\right) \psi_{d} \\
& -2 G_{1}\left(\left\langle\bar{\psi}_{u} \psi_{u}\right\rangle^{2}+\left\langle\bar{\psi}_{d} \psi_{d}\right\rangle^{2}\right)-4 G_{2}\left\langle\bar{\psi}_{u} \psi_{u}\right\rangle\left\langle\bar{\psi}_{d} \psi_{d}\right\rangle,
\end{aligned}
$$

where $u, d$ quark condensates are given as $\left\langle\bar{\psi}_{u} \psi_{u}\right\rangle$ and $\left\langle\bar{\psi}_{d} \psi_{d}\right\rangle$, respectively. The constituent quark masses for $u$ and $d$ quarks in terms of the chiral condensates can be given as

$$
\begin{aligned}
& M_{u}=m_{u}-4 G_{1}\left\langle\bar{\psi}_{u} \psi_{u}\right\rangle-4 G_{2}\left\langle\bar{\psi}_{d} \psi_{d}\right\rangle, \\
& M_{d}=m_{d}-4 G_{1}\left\langle\bar{\psi}_{d} \psi_{d}\right\rangle-4 G_{2}\left\langle\bar{\psi}_{u} \psi_{u}\right\rangle .
\end{aligned}
$$

Generalizing Eq. (48) for two flavor NJL model, the chiral condensate in the presence of magnetic field and chiral chemical potential can be written as

$$
\langle\bar{\psi} \psi\rangle_{B \neq 0}^{\mu_{5} \neq 0}=\sum_{f=u, d}\left\langle\bar{\psi}_{f} \psi_{f}\right\rangle_{B \neq 0}^{\mu_{5} \neq 0}
$$

where

$$
\begin{aligned}
\left\langle\bar{\psi}_{f} \psi_{f}\right\rangle_{B \neq 0}^{\mu_{s} \neq 0}= & -\frac{N_{c}\left|q_{f}\right| B}{(2 \pi)^{2}}\left[\int d p_{z} \frac{M_{f}}{E_{p_{z}, f}^{(0)}}\left[1-f_{F D}\left(E_{p_{z}, f}^{(0)}-\mu\right)-f_{F D}\left(E_{p_{z}, f}^{(0)}+\mu\right)\right]\right. \\
& \left.+\sum_{n=1}^{\infty} \sum_{s} \int d p_{z} \frac{M_{f}}{E_{p_{z}, s, f}^{(n)}}\left[1-f_{F D}\left(E_{p_{z}, s, f}^{(n)}-\mu\right)-f_{F D}\left(E_{p_{z}, s, f}^{(n)}+\mu\right)\right]\right],
\end{aligned}
$$

and the single particle energy of flavor $f$ can be expressed as

$$
\begin{aligned}
E_{p_{z}, f}^{(0)} & =\sqrt{M_{f}^{2}+\left(p_{z}-\mu_{5}\right)^{2}} \quad \text { for } n=0, \\
E_{p_{z}, s, f}^{(n)} & =\sqrt{M_{f}^{2}+\left(\sqrt{p_{z}^{2}+2 n\left|q_{f}\right| B}-s \mu_{5}\right)^{2}} \quad \text { for } n>0 .
\end{aligned}
$$

For vanishing chiral chemical potential $\mu_{5}=0$, the chiral condensate of single flavor can be expressed as

$$
\begin{aligned}
\left\langle\bar{\psi}_{f} \psi_{f}\right\rangle_{B \neq 0}^{\mu_{5}=0}= & -\frac{N_{c}\left|q_{f}\right| B}{(2 \pi)^{2}} \sum_{n=0}^{\infty}\left(2-\delta_{n, 0}\right) \\
& \times \int d p_{z} \frac{M_{0_{f}}}{\epsilon_{p_{z}, f}^{(n)}}\left[1-f_{F D}\left(\epsilon_{p_{z}, f}^{(n)}-\mu\right)\right. \\
& \left.-f_{F D}\left(\epsilon_{p_{z}, f}^{(n)}+\mu\right)\right]
\end{aligned}
$$

and the single particle energies of flavor $f$ can be expressed as

$$
\epsilon_{p_{z}, f}^{(n)}=\sqrt{p_{z}^{2}+M_{0_{f}}^{2}+2 n\left|q_{f}\right| B} .
$$

The first term of the quark condensate as given in Eq. (57) contains divergence and needs to be regularized to derive meaningful results. Usually, in NJL model at vanishing temperature and chemical potential, such integrals are regularized either by a sharp three momentum cutoff [84,102] or a smooth cutoff [105-107]. Effective models like NJL model which are nonrenormalizable have to be complemented with a regularization scheme with the constraint that the physically meaningful results should be eventually independent of the regularization prescription. In the presence of magnetic field, continuous momentum dependence in two spatial dimensions transverse to the direction of magnetic field is replaced by a sum over discretized Landau levels. Hence, a sharp three momentum cutoff in the presence of the magnetic field can suffer from the cutoff artifacts. Instead, of a sharp cutoff, a smooth momentum cutoff was used in Ref. [54] in the context of chiral magnetic effects in the PNJL model to avoid such sharp cutoff artifacts. To regularize the first term in Eq. (57), we follow an elegant procedure that was followed in Refs. [41,42,44,108-110] by adding and subtracting a vacuum (zero field) term to the chiral condensate which is also divergent. This makes the first term of Eq. (57) neatly separated into a zero field vacuum term and a term that is only dependent on the field written in terms of gamma function which is finite. Thus, the 
regularized chiral condensate in the presence of magnetic field at vanishing quark chiral chemical potential is [see Appendix B, Eq. (B13)]

$$
\begin{aligned}
\left\langle\bar{\psi}_{f} \psi_{f}\right\rangle_{B \neq 0}^{\mu_{5}=0}= & -2 N_{c} \int_{|\vec{p}| \leq \Lambda} \frac{d^{3} p}{(2 \pi)^{3}} \frac{M_{0_{f}}}{\sqrt{p^{2}+M_{0_{f}}^{2}}}-\frac{N_{c} M_{0_{f}}\left|q_{f}\right| B}{2 \pi^{2}}\left[x_{0_{f}}\left(1-\ln x_{0_{f}}\right)+\ln \Gamma\left(x_{0_{f}}\right)+\frac{1}{2} \ln \left(\frac{x_{0_{f}}}{2 \pi}\right)\right] \\
& +\frac{N_{c}\left|q_{f}\right| B}{2 \pi^{2}} \sum_{n=0}^{\infty}\left(2-\delta_{n, 0}\right) \int_{-\infty}^{\infty} d p_{z} \frac{M_{0_{f}}}{\epsilon_{p_{z}, f}^{(n)}} f_{F D}\left(\epsilon_{p_{z}, f}^{(n)}\right),
\end{aligned}
$$

where the dimensionless variable $x_{0_{f}}=M_{0_{f}}^{2} / 2\left|q_{f}\right| B$. Scalar condensate as given in Eq. (59) can also be obtained by minimizing the regularized thermodynamic potential using the mean field Lagrangian as given in Eq. (52) in the case of vanishing chiral chemical potential. Solving Eq. (53) using Eq. (59), we get quark masses for vanishing chiral chemical potential with finite magnetic field. This constituent mass will be later used to estimate quark masses at finite chiral chemical potential and finite magnetic field, as discussed in the following subsection.

\section{A. Regularization of chiral condensate in the presence of magnetic field and chiral chemical potential}

Chiral condensate $\left\langle\bar{\psi}_{f} \psi_{f}\right\rangle$ of quark flavor $f$ in the presence of magnetic field and nonzero chiral chemical potential is given as

$$
\begin{aligned}
\left\langle\bar{\psi}_{f} \psi_{f}\right\rangle_{B \neq 0}^{\mu_{5} \neq 0}= & -\frac{N_{c}\left|q_{f}\right| B}{(2 \pi)^{2}}\left[\int d p_{z} \frac{M_{f}}{E_{p_{z}, f}^{(0)}}\left[1-f_{F D}\left(E_{p_{z}, f}^{(0)}-\mu\right)-f_{F D}\left(E_{p_{z}, f}^{(0)}+\mu\right)\right]\right. \\
& \left.+\sum_{n=1}^{\infty} \sum_{s} \int d p_{z} \frac{M_{f}}{E_{p_{z}, s, f}^{(n)}}\left[1-f_{F D}\left(E_{p_{z}, s, f}^{(n)}-\mu\right)-f_{F D}\left(E_{p_{z}, s, f}^{(n)}+\mu\right)\right]\right] \\
= & \left\langle\bar{\psi}_{f} \psi_{f}\right\rangle_{\mathrm{vac}, \mathrm{B} \neq 0}^{\mu_{5} \neq 0}+\left\langle\bar{\psi}_{f} \psi_{f}\right\rangle_{\mathrm{med}, \mathrm{B} \neq 0}^{\mu_{5} \neq 0},
\end{aligned}
$$

where $\left\langle\bar{\psi}_{f} \psi_{f}\right\rangle_{\text {vac,B } \neq 0}^{\mu_{5} \neq 0}$ is zero temperature and zero quark chemical potential part of the chiral condensate, and $\left\langle\bar{\psi}_{f} \psi_{f}\right\rangle_{\text {med,B } \mathrm{B} \neq 0}^{\mu_{5} \neq 0}$ is the medium term at finite temperature and quark chemical potential. $\left\langle\bar{\psi}_{f} \psi_{f}\right\rangle_{\text {vac,B } \neq 00}^{\mu_{5} \neq 0}$ contains divergent integral which has to be regularized to obtain meaningful physical result. To regularize the vacuum part of the chiral condensate for nonvanishing magnetic field and chiral chemical potential, we have not considered the naive regularization with finite cutoff (Traditional Regularization Scheme (TRS)) to remove cutoff artifacts, rather we have considered MSS outlined in Ref. [111]. By adding and subtracting the lowest Landau level term in the zero temperature and zero quark chemical potential part of the chiral condensate for nonvanishing magnetic field and chiral chemical potential, we get (for details, see Appendix C)

$$
\begin{aligned}
\left\langle\bar{\psi}_{f} \psi_{f}\right\rangle_{\mathrm{vac}, \mathrm{B} \neq 0}^{\mu_{5} \neq 0} & =-\frac{N_{c}\left|q_{f}\right| B}{(2 \pi)^{2}} \sum_{n=0}^{\infty} \sum_{s= \pm 1} \int d p_{z} \frac{M_{f}}{E_{p_{z}, s, f}^{(n)}}+\frac{N_{c}\left|q_{f}\right| B}{(2 \pi)^{2}} \int d p_{z} \frac{M_{f}}{E_{p_{z}, f}^{(0)}} \\
& =-\frac{N_{c}\left|q_{f}\right| B}{(2 \pi)^{2}} \sum_{n=0}^{\infty} \sum_{s= \pm 1} \int d p_{z}\left(\frac{1}{\pi}\right) \int_{-\infty}^{\infty} d p_{4} \frac{M_{f}}{p_{4}^{2}+\left(E_{p_{z}, s, f}^{(n)}\right)^{2}}+\frac{N_{c}\left|q_{f}\right| B}{(2 \pi)^{2}} \int d p_{z} \frac{M_{f}}{E_{p_{z}, f}^{(0)}} \\
& =I_{1}+I_{2},
\end{aligned}
$$

where $E_{p_{z}, s, f}^{(n)}=\sqrt{M_{f}^{2}+\left(\sqrt{p_{z}^{2}+2 n\left|q_{f}\right| B}-s \mu_{5}\right)^{2}}$ and $E_{p_{z}, f}^{(0)}=\sqrt{M_{f}^{2}+\left(p_{z}-\mu_{5}\right)^{2}}$. Both integrals $I_{1}$ and $I_{2}$ are not convergent at large momentum; hence, these integrals have to be regularized to get physically meaningful results. In the present investigation, we are using MSS to regularize the integrals $I_{1}$ and $I_{2}$. MSS method has also been applied in the case of finite chiral chemical potential but vanishing magnetic field in Ref. [59]. In the present case, we keep both $B \neq 0$ and $\mu_{5} \neq 0$ and use the same scheme in the following. Integral $I_{1}$ can be regularized by adding and subtracting the similar term with magnetic field $(B)$ but $\mu_{5}=0$, 


$$
\begin{aligned}
\frac{1}{p_{4}^{2}}+\left(E_{p_{z}, s, f}^{(n)}\right)^{2} \\
=\frac{1}{p_{4}^{2}+\left(\epsilon_{p_{z}, f}^{(n)}\right)^{2}}-\frac{1}{p_{4}^{2}+\left(\epsilon_{p_{z}, f}^{(n)}\right)^{2}}+\frac{1}{p_{4}^{2}+\left(E_{p_{z}, s, f}^{(n)}\right)^{2}} \\
=\frac{1}{p_{4}^{2}+\left(\epsilon_{p_{z}, f}^{(n)}\right)^{2}}+\frac{A+2 s \mu_{5} \sqrt{p_{z}^{2}+2 n\left|q_{f}\right| B}}{\left[p_{4}^{2}+\left(\epsilon_{p_{z}, f}^{(n)}\right)^{2}\right]\left[p_{4}^{2}+\left(E_{p_{z}, s, f}^{(n)}\right)^{2}\right.},
\end{aligned}
$$

where $A=M_{0_{f}}^{2}-M_{f}^{2}-\mu_{5}^{2}$ and $\epsilon_{p_{z}, f}^{(n)}=\sqrt{M_{0_{f}}^{2}+p_{z}^{2}+2 n\left|q_{f}\right| B}$. Using the identity given in Eq. (62) twice, we can write the integrand of the integral $I_{1}$, as given in Eq. (61), in the following way:

$$
\begin{aligned}
\frac{1}{p_{4}^{2}+\left(E_{p_{z}, s, f}^{(n)}\right)^{2}}= & \frac{1}{p_{4}^{2}+\left(\epsilon_{p_{z}, f}^{(n)}\right)^{2}}+\frac{A+2 s \mu_{5} \sqrt{p_{z}^{2}+2 n\left|q_{f}\right| B}}{\left(p_{4}^{2}+\left(\epsilon_{p_{z}, f}^{(n)}\right)^{2}\right)^{2}} \\
& +\frac{\left(A+2 s \mu_{5} \sqrt{p_{z}^{2}+2 n\left|q_{f}\right| B}\right)^{2}}{\left(p_{4}^{2}+\left(\epsilon_{p_{z}, f}^{(n)}\right)^{2}\right)^{3}} \\
& +\frac{\left(A+2 s \mu_{5} \sqrt{p_{z}^{2}+2 n\left|q_{f}\right| B}\right)^{3}}{\left(p_{4}^{2}+\left(\epsilon_{p_{z}, f}^{(n)}\right)^{2}\right)^{3}\left(p_{4}^{2}+\left(E_{p_{z}, s, f}^{(n)}\right)^{2}\right)} .
\end{aligned}
$$

Performing $p_{4}$ integration, we obtain (for details, see Appendix B)

$$
I_{1}=I_{1_{\text {quad }}}-\frac{M_{f}\left(M_{0_{f}}^{2}-M_{f}^{2}+2 \mu_{5}^{2}\right)}{2} I_{1_{\log }}+I_{1_{\text {finite }}}+I_{1_{\text {finite } 2}},
$$

where

$$
\begin{gathered}
I_{1_{\text {quad }}}=-\frac{N_{c}\left|q_{f}\right| B}{(2 \pi)^{2}} \sum_{n=0}^{\infty} \sum_{s} \int d p_{z} \frac{M_{f}}{\epsilon_{p_{z}, f}^{(n)}}, \\
I_{1_{\log }}=\frac{N_{c}\left|q_{f}\right| B}{(2 \pi)^{2}} \sum_{n=0}^{\infty} \sum_{s} \int d p_{z} \frac{1}{\left(\epsilon_{p_{z}, f}^{(n)}\right)^{3}}, \\
I_{1_{\text {finitel }}}=-\frac{N_{c}\left|q_{f}\right| B}{(2 \pi)^{2}} \sum_{n=0}^{\infty} \sum_{s} \int d p_{z}\left(\frac{3}{8}\right) \frac{\left(M_{f} A^{2}-4 M_{f} \mu_{5}^{2} M_{0_{f}}^{2}\right)}{\left(\epsilon_{p_{z}, f}^{(n)}\right)^{5}}
\end{gathered}
$$

and

$$
\begin{aligned}
I_{1_{\text {finite2 }}}= & -\frac{N_{c}\left|q_{f}\right| B}{(2 \pi)^{2}}\left(\frac{15}{16}\right) \sum_{n=0}^{\infty} \sum_{s} \int d p_{z} \\
& \times \int_{0}^{1} d x \frac{(1-x)^{2} M_{f}\left(A+2 s \mu_{5} \sqrt{p_{z}^{2}+2 n\left|q_{f}\right| B}\right)^{3}}{\left[\left(\epsilon_{p_{z}, f}^{(n)}\right)^{2}-x\left(A+2 s \mu_{5} \sqrt{p_{z}^{2}+2 n\left|q_{f}\right| B}\right)\right]^{7 / 2}} .
\end{aligned}
$$

The integrals $I_{1_{\text {quad }}}$ and $I_{1_{\log }}$ are divergent at large momentum. On the other hand, $I_{1_{\text {finite }}}$ and $I_{1_{\text {finite } 2}}$ are finite.

In a similar manner, the integral $I_{2}$ in Eq. (61), we obtain

$$
I_{2}=I_{2_{\text {finite }}}+I_{2_{\log }},
$$

where

$$
\begin{aligned}
I_{2_{\text {finite }}}= & \left(\frac{1}{2}\right) \frac{N_{c}\left|q_{f}\right| B}{(2 \pi)^{2}} \int d p_{z} \\
& \times \int_{0}^{1} d x \frac{M_{f}\left(A+2 p_{z} \mu_{5}\right)}{\left[\left(\epsilon_{p_{z}, f}^{(0)}\right)^{2}-x\left(A+2 p_{z} \mu_{5}\right)\right]^{3 / 2}},
\end{aligned}
$$

and

$$
I_{2_{\log }}=\frac{N_{c}\left|q_{f}\right| B}{(2 \pi)^{2}} \int d p_{z} \frac{M_{f}}{\epsilon_{p_{z}, f}^{(0)}} .
$$

Using Eqs. (64) and (69), $\left\langle\bar{\psi}_{f} \psi_{f}\right\rangle_{\mathrm{vac}, \mathrm{B} \neq 0}^{\mu_{5} \neq 0}$ can be expressed as

$$
\begin{aligned}
\left\langle\bar{\psi}_{f} \psi_{f}\right\rangle_{\text {vac, } \mathrm{B} \neq 0}^{\mu_{5} \neq 0}= & -\frac{M_{f}\left(M_{0_{f}}^{2}-M_{f}^{2}+2 \mu_{5}^{2}\right)}{2} I_{1_{\log }}+I_{1_{\text {finitel }}} \\
& +I_{1_{\text {finite2 }}}+I_{2_{\text {finite }}}+I_{\text {quad }},
\end{aligned}
$$

where

$$
\begin{aligned}
I_{\text {quad }}= & I_{1_{\text {quad }}}+I_{2_{\log }} \\
= & \frac{M_{f}}{M_{0_{f}}}\left[-\frac{N_{c}\left|q_{f}\right| B}{(2 \pi)^{2}} \sum_{n=0}^{\infty} \sum_{s} \int d p_{z} \frac{M_{0_{f}}}{\epsilon_{p_{z}, f}^{(n)}}\right. \\
& \left.+\frac{N_{c}\left|q_{f}\right| B}{(2 \pi)^{2}} \int d p_{z} \frac{M_{0_{f}}}{\epsilon_{p_{z}, f}^{(0)}}\right] .
\end{aligned}
$$

Each integral in $I_{\text {quad }}$ is divergent. Using dimensional regularization, it can be regularized to get [see Appendix B, Eqs. (B2) and (B13)] 


$$
\begin{aligned}
I_{\text {quad }}= & \frac{M_{f}}{M_{0_{f}}}\left[-\frac{N_{c}\left|q_{f}\right| B}{(2 \pi)^{2}} \sum_{n=0}^{\infty} \sum_{s} \int d p_{z} \frac{M_{0_{f}}}{\epsilon_{p_{z}, f}^{(n)}}\right. \\
& \left.+\frac{N_{c}\left|q_{f}\right| B}{(2 \pi)^{2}} \int d p_{z} \frac{M_{0_{f}}}{\epsilon_{p_{z}, f}^{(0)}}\right]=I_{\text {quad }}^{\text {field }}+I_{\text {quad }}^{\text {vac }},
\end{aligned}
$$

where

$$
\begin{aligned}
I_{\text {quad }}^{\text {field }}= & -\frac{N_{c} M_{f}\left|q_{f}\right| B}{2 \pi^{2}}\left[x_{0_{f}}\left(1-\ln x_{0_{f}}\right)\right. \\
& \left.+\ln \Gamma\left(x_{0_{f}}\right)+\frac{1}{2} \ln \left(\frac{x_{0_{f}}}{2 \pi}\right)\right]
\end{aligned}
$$

and,

$$
\begin{aligned}
I_{\mathrm{quad}}^{\mathrm{vac}}= & -\frac{N_{c} M_{f}}{2 \pi^{2}}\left[\Lambda \sqrt{M_{0_{f}}^{2}+\Lambda^{2}}\right. \\
& \left.-M_{0_{f}}^{2} \ln \left(\frac{\Lambda+\sqrt{\Lambda^{2}+M_{0_{f}}^{2}}}{M_{0_{f}}}\right)\right] .
\end{aligned}
$$

Similarly, the term $I_{1_{\log }}$ is divergent at large momentum, hence it has to be regularized. Regularization of $I_{1_{\log }}$ can be done using dimensional regularization. In the dimensional regularization scheme [see Appendix B, Eq. (B16)],

$$
\begin{aligned}
I_{1_{\log }} & =\frac{N_{c}\left|q_{f}\right| B}{(2 \pi)^{2}} \sum_{n=0}^{\infty} \sum_{s} \int d p_{z} \frac{1}{\left(\epsilon_{p_{z}, f}^{(n)}\right)^{3}} \\
& =I_{1_{\log }}^{\mathrm{field}}+I_{1_{\log }}^{\mathrm{vac}} .
\end{aligned}
$$

Here,

$$
I_{1_{\log }}^{\text {field }}=-\frac{N_{c}}{2 \pi^{2}}\left[-\ln x_{0_{f}}+\frac{\Gamma^{\prime}\left(x_{0_{f}}\right)}{\Gamma\left(x_{0_{f}}\right)}\right]
$$

and

$$
I_{1_{\log }}^{\mathrm{vac}}=\frac{N_{c}}{\pi^{2}}\left(\ln \left(\frac{\Lambda}{M_{0_{f}}}+\sqrt{1+\frac{\Lambda^{2}}{M_{0_{f}}^{2}}}\right)-\frac{\Lambda}{\sqrt{\Lambda^{2}+M_{0_{f}}^{2}}}\right) .
$$

Hence, the regularized chiral condensate of quark flavor $f$ for finite magnetic field and chiral chemical potential in MSS for vanishing quark chemical potential can be expressed as

$$
\begin{aligned}
\left\langle\bar{\psi}_{f} \psi_{f}\right\rangle_{B \neq 0}^{\mu_{5} \neq 0}= & -\frac{M_{f}\left(M_{0_{f}}^{2}-M_{f}^{2}+2 \mu_{5}^{2}\right)}{2} I_{1_{\text {log }}}+I_{1_{\text {finite } 1}} \\
& +I_{1_{\text {finite } 2}+I_{2_{\text {finite }}}+I_{\text {quad }}} \\
& +\frac{N_{c}\left|q_{f}\right| B}{2 \pi^{2}}\left[\int_{-\infty}^{\infty} d p_{z} \frac{M_{f}}{E_{p_{z}, f}^{(0)}} f_{F D}\left(E_{p_{z}, f}^{(0)}\right)\right. \\
& \left.+\sum_{n=1}^{\infty} \sum_{s} \int_{-\infty}^{\infty} d p_{z} \frac{M_{f}}{E_{p_{z}, s, f}^{(n)}} f_{F D}\left(E_{p_{z}, s, f}^{(n)}\right)\right],
\end{aligned}
$$

where regularized $I_{1_{\log }}$ and $I_{\text {quad }}$ are given in Eqs. (77) and (74), respectively. This makes the expression for $\left\langle\bar{\psi}_{f} \psi_{f}\right\rangle_{B \neq 0}^{\mu_{5} \neq 0}$ finite which we shall use later for the calculation of constituent mass $\left(M_{f}\right)$ for nonvanishing magnetic field and chiral chemical potential. Note that for the estimation of constituent mass $\left(M_{f}\right)$ for nonvanishing magnetic field and chiral chemical potential, one requires constituent mass $M_{0_{f}}$ for nonvanishing magnetic field and vanishing chiral chemical potential, which can be obtained from Eq. (59).

\section{CHIRAL SUSCEPTIBILITY}

The fluctuations and correlations are an important characteristics of any physical system. They provide essential information about the effective d.o.f. and their possible quasiparticle nature. These fluctuations and correlations are connected with susceptibility. Susceptibility is the response of the system to small external force. The chiral susceptibility measures the response of the chiral condensate to the infinitesimal change of the current quark mass. Chiral susceptibility in two flavor NJL model can be defined as

$\chi_{c}=\frac{\partial\langle\bar{\psi} \psi\rangle}{\partial m}=\frac{\partial\left\langle\bar{\psi}_{u} \psi_{u}\right\rangle}{\partial m}+\frac{\partial\left\langle\bar{\psi}_{d} \psi_{d}\right\rangle}{\partial m}=\chi_{c u}+\chi_{c d}$.

Using Eq. (53), we get

$$
\begin{aligned}
\chi_{c u} & =\frac{\partial\left\langle\bar{\psi}_{u} \psi_{u}\right\rangle}{\partial m} \\
& =\frac{\partial\left\langle\bar{\psi}_{u} \psi_{u}\right\rangle}{\partial M_{u}}\left(1-4 G_{1} \frac{\partial\left\langle\bar{\psi}_{u} \psi_{u}\right\rangle}{\partial m}-4 G_{2} \frac{\partial\left\langle\bar{\psi}_{d} \psi_{d}\right\rangle}{\partial m}\right)
\end{aligned}
$$

and

$$
\begin{aligned}
\chi_{c d} & =\frac{\partial\left\langle\bar{\psi}_{d} \psi_{d}\right\rangle}{\partial m} \\
& =\frac{\partial\left\langle\bar{\psi}_{d} \psi_{d}\right\rangle}{\partial M_{d}}\left(1-4 G_{1} \frac{\partial\left\langle\bar{\psi}_{d} \psi_{d}\right\rangle}{\partial m}-4 G_{2} \frac{\partial\left\langle\bar{\psi}_{u} \psi_{u}\right\rangle}{\partial m}\right) .
\end{aligned}
$$


Using Eq. (82), Eq. (83) solving for $\chi_{c u}$ and $\chi_{c d}$, we get

$$
\chi_{c u}=\frac{\partial\left\langle\bar{\psi}_{u} \psi_{u}\right\rangle}{\partial M_{u}} \frac{1-4 G_{2} \chi_{c d}}{1+4 G_{1} \frac{\partial\left\langle\bar{\psi}_{u} \psi_{u}\right\rangle}{\partial M_{u}}}
$$

and

$$
\chi_{c d}=\frac{\partial\left\langle\bar{\psi}_{d} \psi_{d}\right\rangle}{\partial M_{d}} \frac{1-4 G_{2} \chi_{c u}}{1+4 G_{1} \frac{\partial\left\langle\bar{\psi}_{d} \psi_{d}\right\rangle}{\partial M_{d}}} .
$$

Solving Eqs. (84) and (85), we get

$$
\begin{aligned}
& \chi_{c u}=\frac{\partial\left\langle\bar{\psi}_{u} \psi_{u}\right\rangle}{\partial M_{u}}\left(\frac{1+4\left(G_{1}-G_{2}\right) \frac{\partial\left\langle\bar{\psi}_{d} \psi_{d}\right\rangle}{\partial M_{d}}}{\left(1+4 G_{1} \frac{\partial\left\langle\bar{\psi}_{u} \psi_{u}\right\rangle}{\partial M_{u}}\right)\left(1+4 G_{1} \frac{\partial\left\langle\bar{\psi}_{d} \psi_{d}\right\rangle}{\partial M_{d}}\right)-16 G_{2}^{2} \frac{\partial\left\langle\bar{\psi}_{u} \psi_{u}\right\rangle}{\partial M_{u}} \frac{\partial\left\langle\bar{\psi}_{d} \psi_{d}\right\rangle}{\partial M_{d}}}\right), \\
& \chi_{c d}=\frac{\partial\left\langle\bar{\psi}_{d} \psi_{d}\right\rangle}{\partial M_{d}}\left(\frac{1+4\left(G_{1}-G_{2}\right) \frac{\partial\left\langle\bar{\psi}_{u} \psi_{u}\right\rangle}{\partial M_{u}}}{\left(1+4 G_{1} \frac{\partial\left\langle\bar{\psi}_{u} \psi_{u}\right\rangle}{\partial M_{u}}\right)\left(1+4 G_{1} \frac{\partial\left\langle\bar{\psi}_{d} \psi_{d}\right\rangle}{\partial M_{d}}\right)-16 G_{2}^{2} \frac{\partial\left\langle\bar{\psi}_{u} \psi_{u}\right\rangle}{\partial M_{u}} \frac{\partial\left\langle\bar{\psi}_{d} \psi_{d}\right\rangle}{\partial M_{d}}}\right) .
\end{aligned}
$$

It is clear from Eqs. (86) and (87) that to calculate chiral susceptibility for $u$ and $d$ quarks, we have to estimate $\frac{\partial\left\langle\bar{\psi}_{f} \psi_{f}\right\rangle}{\partial M_{f}}$. However, it is important to note that like chiral condensate, chiral susceptibility also contains ultraviolet divergence. Hence, $\frac{\partial\left\langle\bar{\psi}_{f} \psi_{f}\right\rangle}{\partial M_{f}}$ term also has to be regularized to get meaningful results. Using Eq. (59), for vanishing chemical potential $(\mu=0)$ and vanishing chiral chemical potential $\left(\mu_{5}=0\right)$, in the presence of magnetic field, we get

$$
\begin{aligned}
\frac{\partial\left\langle\bar{\psi}_{f} \psi_{f}\right\rangle_{B \neq 0}^{\mu_{5}=0}}{\partial M_{0_{f}}}= & -\frac{2 N_{c}}{(2 \pi)^{3}} \int_{|\vec{p}| \leq \Lambda} d^{3} p\left[\frac{1}{\sqrt{p^{2}+M_{0_{f}}^{2}}}-\frac{M_{0_{f}}^{2}}{\sqrt{\left(p^{2}+M_{0_{f}}^{2}\right)^{3}}}\right] \\
& -\frac{N_{c}\left|q_{f}\right| B}{2 \pi^{2}}\left[x_{0_{f}}\left(1-\ln x_{0_{f}}\right)+\ln \Gamma\left(x_{0_{f}}\right)+\frac{1}{2} \ln \left(\frac{x_{0_{f}}}{2 \pi}\right)\right]-\frac{N_{c} M_{0_{f}}^{2}}{2 \pi^{2}}\left[-\ln x_{0_{f}}+\frac{1}{2 x_{0_{f}}}+\frac{\Gamma^{\prime}\left(x_{0_{f}}\right)}{\Gamma\left(x_{0_{f}}\right)}\right] \\
& +\sum_{n=0}^{\infty} \frac{N_{c}\left|q_{f}\right| B}{\pi^{2}}\left(2-\delta_{n, 0}\right) \int_{0}^{\infty} d p_{z}\left[\frac{1}{\epsilon_{p_{z}, f}^{(n)}} f_{F D}\left(\epsilon_{p_{z}, f}^{(n)}\right)-\frac{M_{0_{f}}^{2}}{\left(\epsilon_{p_{z}, f}^{(n)}\right)^{3}} f_{F D}\left(\epsilon_{p_{z}, f}^{(n)}\right)\right. \\
& \left.-\frac{1}{T}\left(\frac{M_{0_{f}}}{\epsilon_{p_{z}, f}^{(n)}}\right)^{2} f_{F D}\left(\epsilon_{p_{z}, f}^{(n)}\right)\left(1-f_{F D}\left(\epsilon_{p_{z}, f}^{(n)}\right)\right)\right] .
\end{aligned}
$$

$\frac{\partial\left\langle\left.\bar{\psi}_{f} \psi_{f}\right|_{B \neq 0} ^{\mu_{5}=0}\right.}{\partial M_{0_{f}}}$ as given in Eq. (88) is regularized and it can be used to calculate $\chi_{c u}, \chi_{c u}$ and chiral susceptibility $\chi_{c}$ for finite magnetic field, but vanishing chiral chemical potential. To estimate chiral susceptibility at finite magnetic field as well as nonvanishing chiral chemical potential, we have to estimate regularized $\frac{\partial\left\langle\bar{\psi}_{f} \psi_{f}\right\rangle}{\partial M_{f}}$ at finite $B$ and $\mu_{5}$. This regularization has been done using the MSS regularization scheme.

\section{A. Regularization of chiral susceptibility in the presence of magnetic field and chiral chemical potential}

For nonvanishing magnetic field $(B)$ and chiral chemical potential $\left(\mu_{5}\right)$ for $\mu=0$, using Eq. (55), the variation of chiral condensate with constituent quark mass can be written as

$$
\frac{\partial\left\langle\bar{\psi}_{f} \psi_{f}\right\rangle_{B \neq 0}^{\mu_{5} \neq 0}}{\partial M_{f}}=\frac{\partial\left\langle\bar{\psi}_{f} \psi_{f}\right\rangle_{\text {vac, } \mathrm{B} \neq 0}^{\mu_{5} \neq 0}}{\partial M_{f}}+\frac{\partial\left\langle\bar{\psi}_{f} \psi_{f}\right\rangle_{\text {med, } \mathrm{B} \neq 0}^{\mu_{5} \neq 0}}{\partial M_{f}} .
$$


Here, the first term is the "vacuum" term given as

$$
\begin{aligned}
\frac{\partial\left\langle\bar{\psi}_{f} \psi_{f}\right\rangle_{\mathrm{vac}, \mathrm{B} \neq 0}^{\mu_{\mathrm{s}} \neq 0}}{\partial M_{f}}= & -\frac{N_{c}\left|q_{f}\right| B}{(2 \pi)^{2}} \sum_{n=0}^{\infty} \sum_{s= \pm 1} \int d p_{z} \frac{1}{E_{p_{z}, s, f}^{(n)}}+\frac{N_{c}\left|q_{f}\right| B}{(2 \pi)^{2}} \int d p_{z} \frac{1}{E_{p_{z}, f}^{(0)}} \\
& +\frac{N_{c}\left|q_{f}\right| B}{(2 \pi)^{2}} \sum_{n=0}^{\infty} \sum_{s= \pm 1} \int d p_{z} \frac{M_{f}^{2}}{\left(E_{p_{z}, s, f}^{(n)}\right)^{3}}-\frac{N_{c}\left|q_{f}\right| B}{(2 \pi)^{2}} \int d p_{z} \frac{M_{f}^{2}}{\left(E_{p_{z}, f}^{(0)}\right)^{3}} \\
= & \mathbf{I}_{1}+\mathbf{I}_{2}+\mathbf{I}_{3}+\mathbf{I}_{4},
\end{aligned}
$$

and the medium dependent term is given as

$$
\begin{aligned}
\frac{\partial\left\langle\bar{\psi}_{f} \psi_{f}\right\rangle_{\text {med, } \mathrm{B} \neq 0}^{\mu_{5} \neq 0}}{\partial M_{f}}= & \frac{N_{c}\left|q_{f}\right| B}{(2 \pi)^{2}} \int d p_{z} \frac{1}{E_{p_{z}, f}^{(0)}}\left(2 f_{F D}\left(E_{p_{z}, f}^{(0)}\right)\right)-\frac{N_{c}\left|q_{f}\right| B}{(2 \pi)^{2}} \int d p_{z} \frac{M_{f}^{2}}{\left(E_{p_{z}, f}^{(0)}\right)^{3}}\left(2 f_{F D}\left(E_{p_{z}, f}^{(0)}\right)\right) \\
& -\frac{N_{c}\left|q_{f}\right| B}{(2 \pi)^{2}} \int d p_{z} \frac{M_{f}^{2}}{\left(E_{p_{z}, f}^{(0)}\right)^{2}}\left(\frac{2}{T}\right) f_{F D}\left(E_{p_{z}, f}^{(0)}\right)\left(1-f_{F D}\left(E_{p_{z}, f}^{(0)}\right)\right) \\
& +\frac{N_{c}\left|q_{f}\right| B}{(2 \pi)^{2}} \sum_{n=1}^{\infty} \sum_{s= \pm 1} \int d p_{z} \frac{1}{E_{p_{z}, s, f}^{(n)}}\left(2 f_{F D}\left(E_{p_{z}, s, f}^{(n)}\right)\right)-\frac{N_{c}\left|q_{f}\right| B}{(2 \pi)^{2}} \sum_{n=1}^{\infty} \sum_{s= \pm 1} \int d p_{z} \frac{M_{f}^{2}}{\left(E_{p_{z}, s, f}^{(n)}\right)^{3}}\left(2 f_{F D}\left(E_{p_{z}, s, f}^{(n)}\right)\right) \\
& -\frac{N_{c}\left|q_{f}\right| B}{(2 \pi)^{2}} \sum_{n=1}^{\infty} \sum_{s= \pm 1} \int d p_{z} \frac{M_{f}^{2}}{\left(E_{p_{z}, s, f}^{(n)}\right)^{2}}\left(\frac{2}{T}\right) f_{F D}\left(E_{p_{z}, s, f}^{(n)}\right)\left(1-f_{F D}\left(E_{p_{z}, s, f}^{(n)}\right)\right) .
\end{aligned}
$$

The medium dependent term is convergent and does not need any regularization. The "vacuum" term, on the other hand, the integrals, $\mathbf{I}_{1}, \mathbf{I}_{2}$, and $\mathbf{I}_{3}$ are divergent and need regularization. We perform the MSS scheme as was done for the chiral condensate. The regularized $\frac{\partial\left\langle\bar{\psi}_{f} \psi_{f}\right\rangle_{\text {vac, } B \neq 0}^{\mu_{f} \neq 0}}{\partial M_{f}}$ can be expressed as [see Appendix D, Eq. (D13)]

$$
\frac{\partial\left\langle\bar{\psi}_{f} \psi_{f}\right\rangle_{\mathrm{vac}, \mathrm{B} \neq 0}^{\mu_{\mathrm{f}} \neq 0}}{\partial M_{f}}=-\left(\frac{M_{0_{f}}^{2}-M_{f}^{2}+2 \mu_{5}^{2}}{2}\right) \mathbf{I}_{1, \log }+\mathbf{I}_{1, \text { finite } 1}+\mathbf{I}_{1, \text { finite } 2}+\mathbf{I}_{2, \text { finite }}+\mathbf{I}_{3, \text { finite }}+\mathbf{I}_{\text {finite }}+\mathbf{I}_{\text {quad }}+\mathbf{I}_{\text {log }},
$$

where regularized $\mathbf{I}_{\text {quad }}, \mathbf{I}_{\log }, \mathbf{I}_{1, \log }$ can be expressed as (see Appendix D, Eqs. (D15)-(D17)]

$$
\begin{gathered}
\mathbf{I}_{\text {quad }}=-\frac{N_{c}\left|q_{f}\right| B}{(2 \pi)^{2}} \sum_{n=0}^{\infty} \sum_{s= \pm 1} \int d p_{z} \frac{1}{\epsilon_{p_{z}, f}^{(n)}}+\frac{N_{c}\left|q_{f}\right| B}{(2 \pi)^{2}} \int d p_{z} \frac{1}{\epsilon_{p_{z}, f}^{(0)}} \\
=-\frac{N_{c}\left|q_{f}\right| B}{2 \pi^{2}}\left[x_{0_{f}}\left(1-\ln x_{0_{f}}\right)+\ln \Gamma\left(x_{0_{f}}\right)+\frac{1}{2} \ln \left(\frac{x_{0_{f}}}{2 \pi}\right)\right]-\frac{N_{c}}{2 \pi^{2}}\left[\Lambda \sqrt{\Lambda^{2}+M_{0_{f}}^{2}}-M_{0_{f}}^{2} \ln \left(\frac{\Lambda+\sqrt{\Lambda^{2}+M_{0_{f}}}}{M_{0_{f}}}\right)\right], \\
\mathbf{I}_{\log }=\frac{N_{c}\left|q_{f}\right| B}{(2 \pi)^{2}} \sum_{n=0}^{\infty} \sum_{s= \pm 1} \int d p_{z} \frac{M_{f}^{2}}{\left(\epsilon_{p_{z}, f}^{(n)}\right)^{3}}-\frac{N_{c}\left|q_{f}\right| B}{(2 \pi)^{2}} \int d p_{z} \frac{M_{f}^{2}}{\left(\epsilon_{p_{z}, f}^{(0)}\right)^{3}} \\
=-\frac{N_{c} M_{f}^{2}}{2 \pi^{2}}\left[-\ln x_{0_{f}}+\frac{1}{2 x_{0_{f}}}+\frac{\Gamma^{\prime}\left(x_{0_{f}}\right)}{\Gamma\left(x_{0_{f}}\right)}\right]+\frac{N_{c} M_{f}^{2}}{\pi^{2}}\left[\ln \left(\frac{\Lambda+\sqrt{\Lambda^{2}+M_{0_{f}}^{2}}}{M_{0_{f}}}\right)-\frac{\Lambda}{\sqrt{\Lambda^{2}+M_{0_{f}}^{2}}}\right]
\end{gathered}
$$




$$
\begin{aligned}
\mathbf{I}_{1, \log } & =\frac{N_{c}\left|q_{f}\right| B}{(2 \pi)^{2}} \sum_{n=0}^{\infty} \sum_{s= \pm 1} \int d p_{z} \frac{1}{\left(\epsilon_{p_{z}, f}^{(n)}\right)^{3}} \\
& =-\frac{N_{c}}{2 \pi^{2}}\left[-\ln x_{0_{f}}+\frac{\Gamma^{\prime}\left(x_{0_{f}}\right)}{\Gamma\left(x_{0_{f}}\right)}\right]+\frac{N_{c}}{\pi^{2}}\left[\ln \left(\frac{\Lambda+\sqrt{\Lambda^{2}+M_{0_{f}}^{2}}}{M_{0_{f}}}\right)-\frac{\Lambda}{\sqrt{\Lambda^{2}+M_{0_{f}}^{2}}}\right],
\end{aligned}
$$

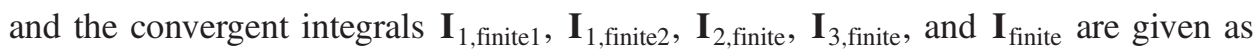

$$
\begin{gathered}
\mathbf{I}_{1, \text { finite } 1}=-\frac{N_{c}\left|q_{f}\right| B}{(2 \pi)^{2}} \sum_{n=0}^{\infty} \sum_{s= \pm 1} \int d p_{z}\left(\frac{3}{8}\right) \frac{A^{2}-4 \mu_{5}^{2} M_{f}^{2}}{\left(\epsilon_{p_{z}, f}^{(n)}\right)^{5}} \\
\mathbf{I}_{1, \text { finite } 2}=-\frac{N_{c}\left|q_{f}\right| B}{(2 \pi)^{2}}\left(\frac{15}{16}\right) \sum_{n=0}^{\infty} \sum_{s= \pm 1} \int d p_{z} \int_{0}^{1} d x \frac{(1-x)^{2}\left(A+2 s \mu_{5} \sqrt{p_{z}^{2}+2 n\left|q_{f}\right| B}\right)^{3}}{\left[\left(\epsilon_{p_{z}, f}^{(n)}\right)^{2}-x\left(A+2 s \mu_{5} \sqrt{p_{z}^{2}+2 n\left|q_{f}\right| B}\right)\right]^{7 / 2}} \\
\mathbf{I}_{2, \text { finite }}=\left(\frac{1}{2}\right) \frac{N_{c}\left|q_{f}\right| B}{(2 \pi)^{2}} \int d p_{z} \int_{0}^{1} d x \frac{A+2 p_{z} \mu_{5}}{\left[\left(\epsilon_{p_{z}, f}^{(0)}\right)^{2}-x\left(A+2 p_{z} \mu_{5}\right)\right]^{3 / 2}},
\end{gathered}
$$

$\mathbf{I}_{3, \text { finite }}=\frac{N_{c}\left|q_{f}\right| B}{(2 \pi)^{2}} \sum_{n=0}^{\infty} \sum_{s= \pm 1} \int d p_{z} M_{f}^{2}\left(\frac{1}{\left(E_{p_{z}, s, f}^{(n)}\right)^{3}}-\frac{1}{\left(\epsilon_{p_{z}, f}^{(n)}\right)^{3}}\right)$,

$\mathbf{I}_{\text {finite }}=-\frac{N_{c}\left|q_{f}\right| B}{(2 \pi)^{2}} \int d p_{z} M_{f}^{2}\left(\frac{1}{\left(E_{p_{z}, f}^{(0)}\right)^{3}}-\frac{1}{\left(\epsilon_{p_{z}, f}^{(0)}\right)^{3}}\right)$.

For nonvanishing magnetic field and chiral chemical potential Eq. (91), Eq. (92) along with Eqs. (86) and (87), can be used to calculate chiral susceptibility $\left(\chi_{c}\right)$.

\section{RESULTS}

Let us note that the Lagrangian as given in Eq. (51) has the following parameters, two couplings $G_{1}, G_{2}$, the three momentum cutoff $\Lambda$, and the current quark masses $m_{u}$ and $m_{d}$. To study the effects of flavor mixing, the couplings $G_{1}$ and $G_{2}$ are parametrized as $G_{2}=\alpha g$, $G_{1}=(1-\alpha) g$ [102]. The extent of flavor mixing is controlled by $\alpha$. For the numerical studies, we take the parameters $m_{u}=m_{d}=6 \mathrm{MeV}$, the three momentum cutoff $\Lambda=590 \mathrm{MeV}$, and the scalar coupling $g=2.435 / \Lambda^{2}$. For these values of the parameters, pion vacuum mass is 140.2 MeV, pion decay constant is $92.6 \mathrm{MeV}$, and the quark condensates are $\left\langle\bar{\psi}_{u} \psi_{u}\right\rangle=\left\langle\bar{\psi}_{d} \psi_{d}\right\rangle=(-241.5) \mathrm{MeV}^{3}$. This parameter set also leads to a vacuum constituent quark mass $400 \mathrm{MeV}$. It may be relevant here to mention that in the absence of magnetic field the two condensates $\left\langle\bar{\psi}_{u} \psi_{u}\right\rangle=\left\langle\bar{\psi}_{d} \psi_{d}\right\rangle$ and therefore the gap equation (53) depends upon the sum of the two couplings $\left(G_{1}+G_{2}\right)$ which is independent of $\alpha$. Thus, the masses $M_{u}$ and $M_{d}$ are the same and do not depend upon $\alpha$ in the absence of magnetic field.

Next, we discuss about choosing the parameter $\alpha$. One can fix the parameter $\alpha$ from the mass of the isoscalar pseudoscalar particle that arises in the spectrum because of breaking of $U(1)_{A}$ symmetry. In a two flavor case, this meson can be identified with the $\eta$ meson. The mass of $\eta$ meson can be given approximately by [103]

$$
m_{\eta}^{2}=m_{\pi}^{2}+\frac{G_{2} M^{2}}{\left(G_{1}^{2}-G_{2}^{2}\right) f_{\pi}^{2}}
$$

Clearly, for $\alpha=0.5$, the $\eta$ meson disappears from the spectrum. With the physical mass of the $\eta$ meson $\left(m_{\eta}=547.8 \mathrm{MeV}\right)$, the above equation leads to a value of $\alpha \simeq 0.09$. On the other hand, a description of $\eta$ meson without strange quarks is not realistic and therefore a better way to fix $\alpha$ is from the three flavor NJL model in which case the determinant interaction becomes a six fermion interaction and leads to $\eta-\eta^{\prime}$ splitting. In such a case, e.g., the gap equation for $M_{u}$ becomes [102]

$$
M_{u}=m_{u}-4 G\left\langle\bar{\psi}_{u} \psi_{u}\right\rangle+2 K\left\langle\bar{\psi}_{s} \psi_{s}\right\rangle\left\langle\bar{\psi}_{d} \psi_{d}\right\rangle
$$

Comparing the constituent quark mass as given in Eq. (53), we can identify $G_{1}=G$ and $G_{2}=-\frac{1}{2} K \phi_{s}$, 
where $\phi_{s} \equiv\left\langle\bar{\psi}_{s} \psi_{s}\right\rangle$ is the strange quark condensate. Thus, using the strange quark condensate, we can express $\alpha$ as [102]

$$
\alpha=\frac{-\frac{1}{2} K \phi_{s}}{G-\frac{1}{2} K \phi_{s}} .
$$

The parameters $G, K,\left\langle\bar{\psi}_{s} \psi_{s}\right\rangle$ are fixed from fitting the masses of the pseudoscalar octet. In particular, the determinant interaction parameter $K$ is fixed from the $\eta-\eta^{\prime}$ mass difference. Even in such cases, the value of $\alpha$ can vary about $25 \%-30 \%$ (i.e., from $\alpha=0.21$ to $\alpha=0.16$ ) depending upon the parametrization chosen. This wide variation in $\alpha$ has to do with the different ways $\eta^{\prime}$ is treated in the model. Since NJL model does not confine and $M_{\eta^{\prime}}$ lies above, the threshold for $q \bar{q}$ decays with an unphysical imaginary part of the corresponding polarization diagram. This is an unavoidable feature of NJL model and leaves an uncertainty that is reflected in difference in the parameters of the determinant interaction. Further, it may be mentioned here that, in a different context of spontaneous $C P$ violation in strong interactions, in Ref. [112] it has been argued that $0 \leq \alpha \leq 0.5$ so that spontaneous parity violation is not there for QCD at zero temperature and density for $\theta=0$ in accordance with Vafa-Witten theorem. In the present work, we have considered the cases when $\alpha=0$, i.e., no flavor mixing, $\alpha=0.5$ when both the couplings are same and a value for $\alpha=0.15$ between these two limits to examine the effects of instanton induced flavor mixing interaction in the presence of magnetic field that breaks the isospin symmetry.

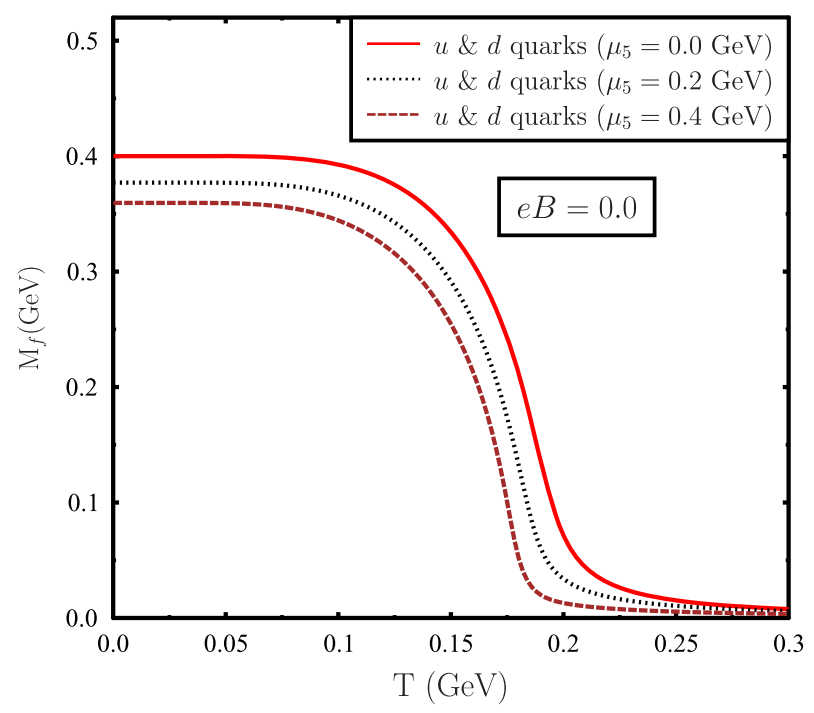

In Fig. 1, we show the variation of constituent quark masses and the associated chiral susceptibility as a function of temperature $(T)$ for different values of chiral chemical potential $\left(\mu_{5}\right)$ and for vanishing magnetic field. For zero magnetic field $\left\langle\bar{\psi}_{u} \psi_{u}\right\rangle=\left\langle\bar{\psi}_{d} \psi_{d}\right\rangle$, hence the masses of the $u$ and $d$ quarks remain same. From the left plot in Fig. 1, we can see that the constituent mass decreases with increasing chiral chemical potential. This decreasing behavior of the constituent quark mass with $\mu_{5}$ is in contrast with other calculations [57,95]. In contrast to Ref. [57], where the condensates increase with $\mu_{5}$ at lower temperature and decrease with $\mu_{5}$ at a higher temperature, we find the scalar condensate always decreases with $\mu_{5}$. Further, we also observe that the chiral transition is a smooth crossover as in Ref. [95] and no first order phase transition is seen even for $\mu_{5}$ as large as $0.4 \mathrm{GeV}$ unlike in Ref. [57]. It ought to be mentioned here that while the vacuum mass satisfies a gap equation with a cutoff in the three momentum, for the thermal contribution no such cutoff was used, similar to Refs. [57,113], as the distribution functions make the corresponding contribution convergent.

The right plot in Fig. 1 shows the chiral susceptibility for vanishing quark chemical potential and magnetic field. Peak in the chiral susceptibility plot shows the chiral transition temperature. Using Eqs. (86) and (87), it can be shown that $\chi_{c u}=\chi_{c d}$ for vanishing magnetic field. Hence, the variation of total chiral susceptibility $\left(\chi_{c}\right)$ with temperature shows only one peak. This behavior of chiral transition temperature decreasing with $\mu_{5}$ is similar to Ref. [54]. Further the height of the peak decreases with $\mu_{5}$ and we do not observe any sharp peak indicative of a first order transition. Absence of a first order transition with

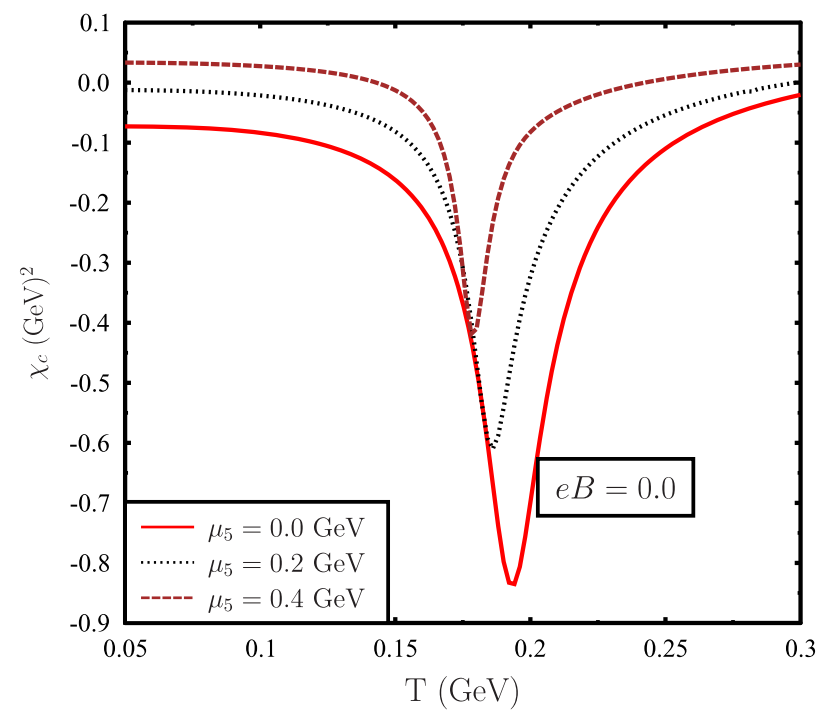

FIG. 1. Left plot: variation of constituent quark mass $M_{u}=M_{d}$ with temperature $(T)$ for zero magnetic field but for various values of chiral chemical potential. Right plot: variation of chiral susceptibility $\chi_{c}$ with temperature $(T)$ for zero magnetic field but with different values of chiral chemical potential. Prominent peak in the chiral susceptibility plot shows the chiral transition temperature. From the left plot, it is clear that with increasing chiral chemical potential $\left(\mu_{5}\right)$ constituent mass decreases. From the susceptibility plot, it is clear that transition temperature decreases with chiral chemical potential. 
large $\mu_{5}$ was also observed in Ref. [95]. However, in the presence of magnetic field, in general $\chi_{c u}$ can be different from $\chi_{c d}$ and variation of total chiral susceptibility $\chi_{c}$ with temperature can show multiple peaks. Results for nonvanishing magnetic field will be shown later. From the right plot in Fig. 1, it is clear that with increasing chiral chemical potential $\left(\mu_{5}\right)$ chiral transition temperature decreases. We would like to mention here that in Ref. [57] for vanishing magnetic field, an opposite behavior regarding chiral transition temperature was observed, i.e., $T_{c}$ increases with $\mu_{5}$. However, the parameters of the NJL model chosen were different compared to the parameters taken here or in Ref. [102]. We have also verified that taking parameters of Ref. [57] $T_{c}$ increases with $\mu_{5}$.

It may be relevant here to compare this behavior of $T_{c}$ with $\mu_{5}$. Such a decreasing behavior of $T_{c}$ with $\mu_{5}$ was also observed in PNJL model; however, the nature of the transition was a first order transition at some critical value of chiral chemical potential [54]. On the other hand, a nonlocal NJL analysis showed the critical temperature to increase with $\mu_{5}$ [95]. A careful analysis in Ref. [57] shows different behavior of $T_{c}$ with $\mu_{5}$. In Ref. [57], it has been shown that if a cutoff is given to the thermal part also then $T_{c}$ increases with $\mu_{5}$ while not giving any cutoff decreases $T_{c}$ with $\mu_{5}$. On the other hand, we have applied here a medium separation scheme to remove cutoff artifact as was done in Refs. $[59,98,99]$. However, our result for vanishing magnetic field showed a opposite behavior, i.e., $T_{c}$ decreases with $\mu_{5}$. It turns out that the behavior of $T_{c}$ with $\mu_{5}$ depends upon the parameter chosen. A stronger scalar coupling as we have taken leads to $T_{c}$ decreasing with $\mu_{5}$, while a weaker scalar coupling shows a mild increase in $T_{c}$ with $\mu_{5}$ [59]. We therefore feel a deeper understanding is still required to understand the opposite behavior of $T_{c}$ with $\mu_{5}$ with change in the scalar coupling. With the parameters considered here, while the behavior of $T_{c}$ decreasing with $\mu_{5}$ is consistent with Ref. [57], the transition itself seems to be a smooth crossover leading to the absence of a critical point in the $\left(\mu_{5}, T\right)$ plane of the phase diagram $[57,95]$.

In Fig. 2, we show the variation of constituent quark masses $M_{u}$ and $M_{d}$ with temperature for vanishing chiral chemical potential and with finite magnetic field for different values of $\alpha$. From this figure, it is clear that at nonvanishing magnetic field constituent quark mass increases. At vanishing magnetic field, constituent mass of $u$ and $d$ quarks is the same. Although in the presence of magnetic field, quark condensates $\left\langle\bar{\psi}_{u} \psi_{u}\right\rangle \neq\left\langle\bar{\psi}_{d} \psi_{d}\right\rangle$, but for $\alpha=0.5$ the quark masses $M_{u}=M_{d}$. This is because for $\alpha=0.5$, constituent quark mass is $M_{f}=m-2 g\left(\left\langle\bar{\psi}_{u} \psi_{u}\right\rangle+\right.$ $\left.\left\langle\bar{\psi}_{d} \psi_{d}\right\rangle\right)$, as can be seen from Eq. (53). On the other hand, for $\alpha \neq 0.5$ quark masses, $M_{u}$ and $M_{d}$ are not the same. The difference between $M_{u}$ and $M_{d}$ increases with decrease in the value of $\alpha$ and this difference is largest when $\alpha=0.0$. $\alpha=0.0$ corresponds to the case when there is no flavor

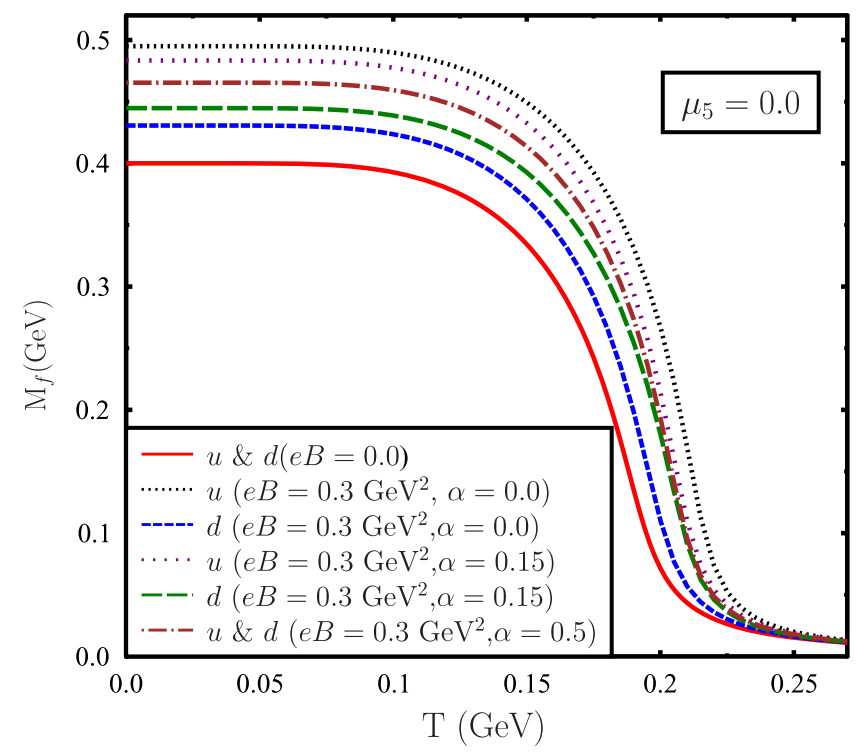

FIG. 2. Variation of constituent quark masses $M_{u}$ and $M_{d}$ with temperature for vanishing chiral chemical potential but with finite magnetic field for different values of $\alpha$. For vanishing magnetic field, $M_{u}$ and $M_{d}$ are same. Note that in the presence of magnetic field, for $\alpha=0.5$, although $\left\langle\bar{\psi}_{u} \psi_{u}\right\rangle \neq\left\langle\bar{\psi}_{d} \psi_{d}\right\rangle$, but the constituent quark masses $M_{u}=M_{d}$. However, for $\alpha \neq 0.5$, the constituent quark masses $M_{u} \neq M_{d}$ in the presence of magnetic field. $\alpha=$ 0.0 corresponds to the case when there is no flavor mixing interaction, and $\alpha=0.5$ corresponds to maximal flavor mixing.

mixing interaction, and $\alpha=0.5$ corresponds to maximal flavor mixing. It is important to note that for vanishing magnetic field, flavor mixing interaction does not affect the quark masses. Only in the presence of magnetic field when $\left\langle\bar{\psi}_{u} \psi_{u}\right\rangle \neq\left\langle\bar{\psi}_{d} \psi_{d}\right\rangle$, flavor mixing interaction affects the constituent quark masses $M_{u}$ and $M_{d}$ significantly.

In Fig. 3, we show the variation of constituent quark masses $M_{u}$ and $M_{d}$ and the associated total chiral susceptibility, with temperature for vanishing chiral chemical potential and with different values of magnetic field for $\alpha=0.5$. It has been already mentioned that for $\alpha=0.5$ even in the presence of magnetic field $M_{u}=M_{d}$. From the left plot in Fig. 3, it is clear that with increasing magnetic field constituent quark mass increases. On the other hand, from the right plot in Fig. 3, it is clear that chiral transition temperature increases with increasing magnetic field.

In Fig. 4, we show the variation of constituent quark masses $M_{u}$ and $M_{d}$ and the associated total chiral susceptibility, with temperature for vanishing chiral chemical potential and with different values of magnetic field for $\alpha=0.0$. For $\alpha=0.0$, there is no flavor mixing. From the left plot, it is clear that at finite magnetic field $M_{u} \neq M_{d}$. This is because in the presence of magnetic field $u$ and $d$ quark condensates are different and in the absence of flavor mixing for $\alpha=0.0, M_{u}$ is independent of $\left\langle\bar{\psi}_{d} \psi_{d}\right\rangle$. Similarly, $M_{d}$ is independent of $\left\langle\bar{\psi}_{u} \psi_{u}\right\rangle$ for $\alpha=0.0$. From the right plot in Fig. 4, it is clear that chiral transition 

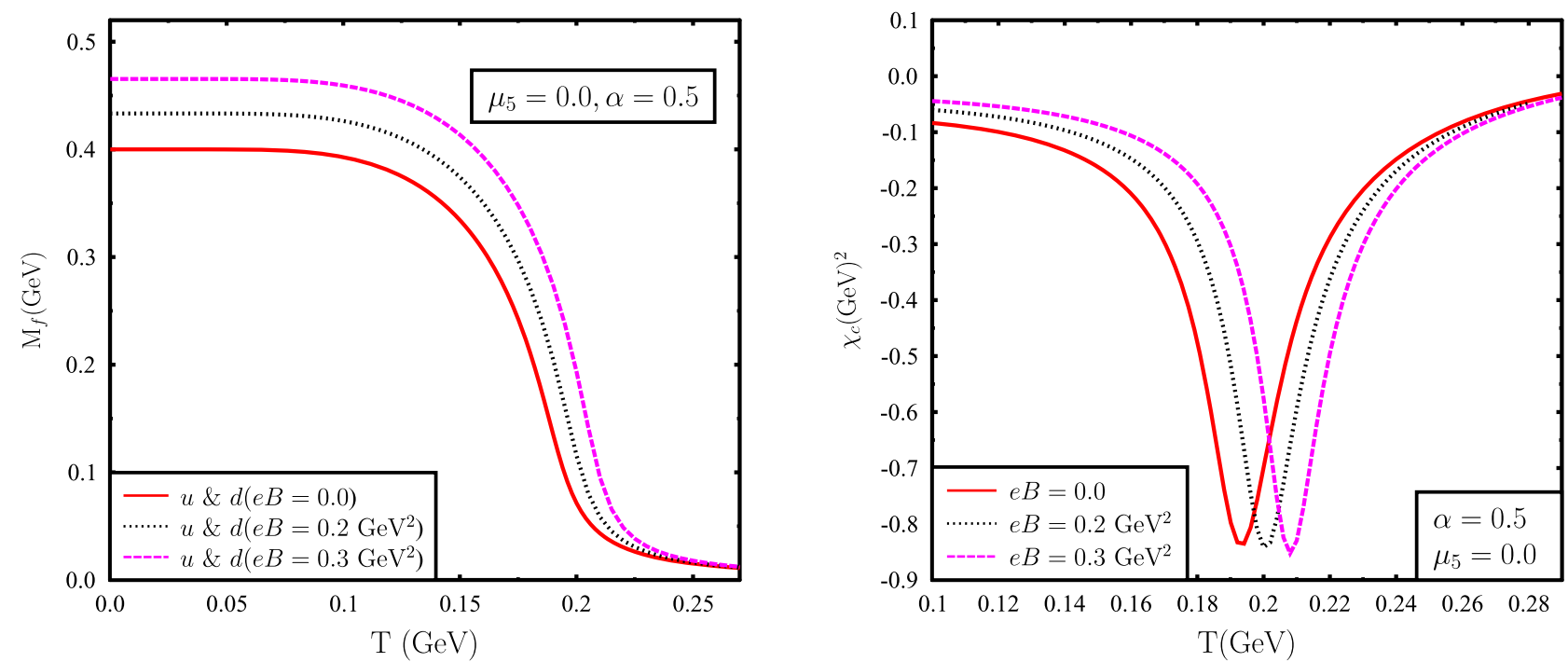

FIG. 3. Left plot: variation of constituent quark mass $M_{u}$ and $M_{d}$, with temperature for vanishing chiral chemical potential, but with different values of magnetic field for $\alpha=0.5$. Right plot: variation of chiral susceptibility $\chi_{c}$ with temperature $(T)$ for vanishing chiral chemical potential, but with different values of magnetic field for $\alpha=0.5$. From the left plot, it is clear that with increasing magnetic field constituent mass $M_{u}=M_{d}$ increases. From the susceptibility plot, it is clear that transition temperature increases with magnetic field.

temperature increases with increasing magnetic field. However, it is important to mention that unlike the case when $\alpha=0.5$, in this case the susceptibility plot shows two distinct peaks at relatively large magnetic field values. In fact, these two peaks are associated with $u$ and $d$ quarks, which have been shown in Fig. 5. In the left plot of Fig. 5, we show $\chi_{c u}, \chi_{c d}$, and $\chi_{c}$ for $e B=0.4 \mathrm{GeV}^{2}$ and $\alpha=0.0$. On the other hand, in the right plot of Fig. 5, we show $\chi_{c u}$,

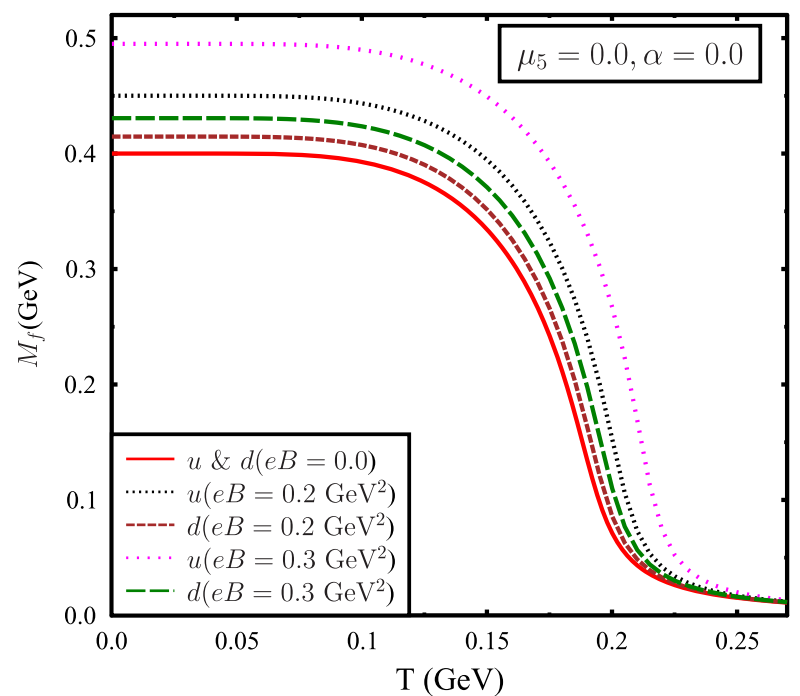

$\chi_{c d}$, and $\chi_{c}$ for $e B=0.4 \mathrm{GeV}^{2}$ and $\alpha=0.5$. From the left plot in Fig. 5, it is clear that for $\alpha=0.0$, i.e., in the absence of flavor mixing, at relatively large magnetic field, chiral susceptibility $\chi_{c}$ shows two distinct peaks. These two peaks are associated with $u$ and $d$ quarks. At relatively large magnetic field with $\alpha=0.0$, chiral restoration of $d$ quark happens at relatively low temperature with respect to the $u$ quarks. This is due to the fact that at nonzero magnetic field

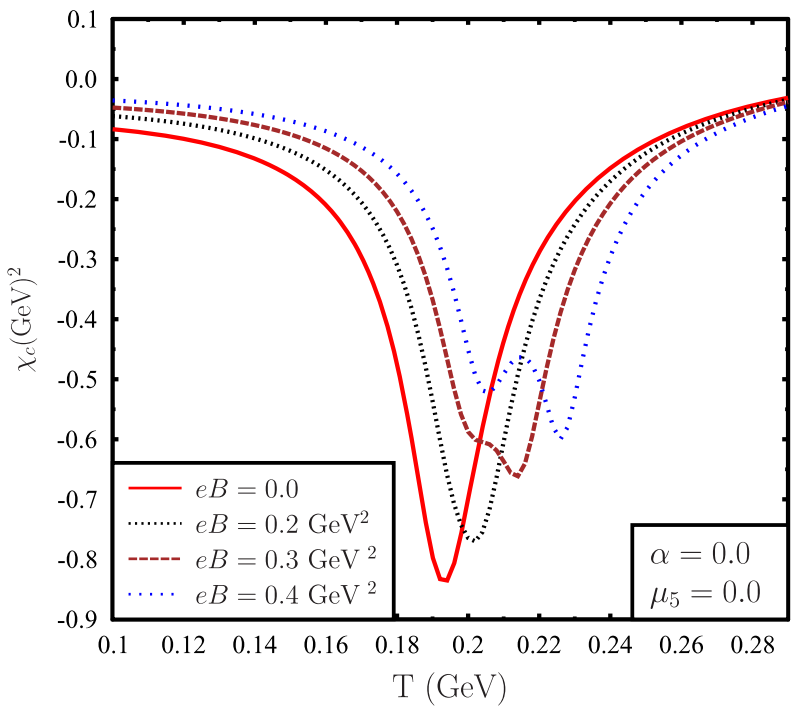

FIG. 4. Left plot: variation of constituent quark mass $M_{u}$ and $M_{d}$, with temperature for vanishing chiral chemical potential, but with different values of magnetic field for $\alpha=0.0$. Right plot: variation of chiral susceptibility $\chi_{c}$ with temperature $(T)$ for vanishing chiral chemical potential, but with different values of magnetic field for $\alpha=0.0$. From the left plot, it is clear that with increasing magnetic field constituent mass increases. From the susceptibility plot, it is clear that transition temperature increases with magnetic field. In the right plot, we can observe two distinct peaks at relatively large magnetic fields. 

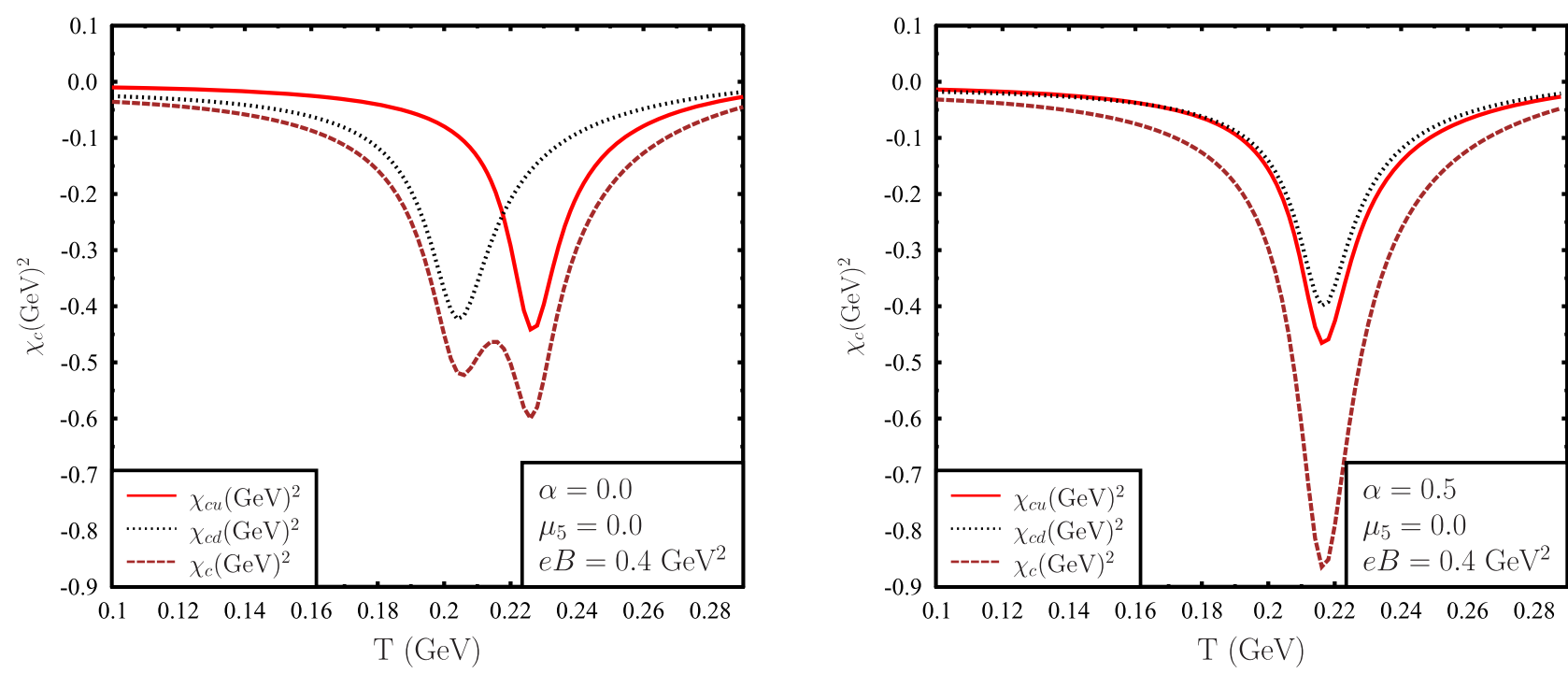

FIG. 5. Left plot: variation of $\chi_{c u}, \chi_{c d}$, and $\chi_{c}$ with temperature at vanishing chiral chemical potential for $e B=0.4 \mathrm{GeV}^{2}$ and $\alpha=0.0$. Right plot: variation of $\chi_{c u}, \chi_{c d}$, and $\chi_{c}$ with temperature at vanishing chiral chemical potential for $e B=0.4 \mathrm{GeV}^{2}$ and $\alpha=0.5$. From the left plot, it is clear that chiral susceptibility shows two distinct peaks at large magnetic field. This is due to the fact that at large magnetic field, difference between $M_{u}$ and $M_{d}$ is large. On the other hand, the right plot shows that for $\alpha=0.5,\left\langle\bar{\psi}_{u} \psi_{u}\right\rangle \neq\left\langle\bar{\psi}_{d} \psi_{d}\right\rangle, \chi_{c u}$ and $\chi_{c d}$ show peak at same temperature. Hence, for $\alpha=0.5$, at finite magnetic field, chiral transition temperature for $u$ and $d$ quarks is the same.

$M_{u}>M_{d}$, as can be seen in Fig. 4. On the other hand, from the right plot in Fig. 5, we can see that, although $\left\langle\bar{\psi}_{u} \psi_{u}\right\rangle \neq\left\langle\bar{\psi}_{d} \psi_{d}\right\rangle, \chi_{c u}$ and $\chi_{c d}$ show peak at same temperature. Hence, for $\alpha=0.5$, at finite magnetic field, chiral transition temperature for $u$ and $d$ quarks is the same.

Finally, in Fig. 6, we show the variation of quark constituent masses $M_{u}$ and $M_{d}$ and the associated susceptibilities with temperature for finite magnetic field and finite chiral chemical potential for $\alpha=0.5$. Behavior of quark constituent masses and the chiral susceptibilities with temperature are similar for other values of $\alpha$. The left plot in Fig. 6 shows that with increasing value of chiral chemical potential and for finite magnetic field constituent quark mass decreases.
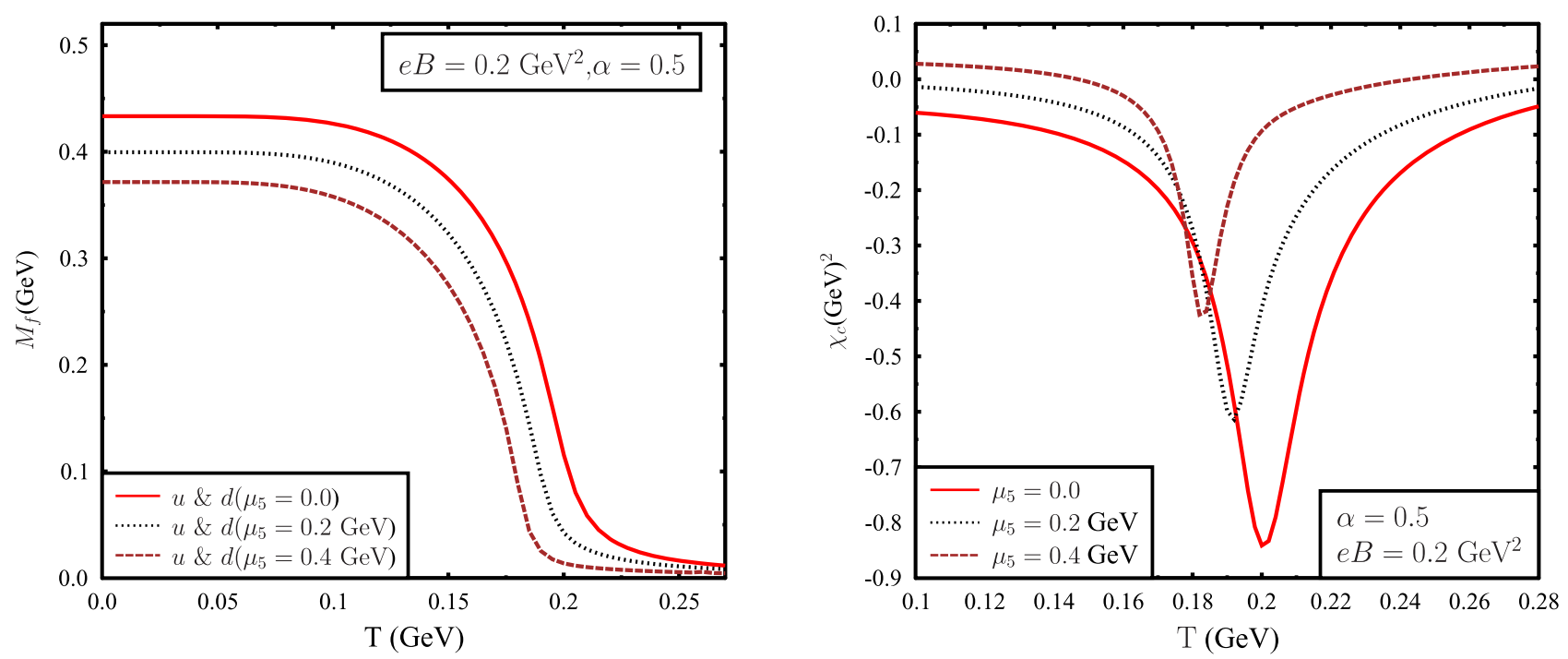

FIG. 6. Left plot: variation of constituent quark mass $M_{u}=M_{d}$, with temperature for finite magnetic field and finite chiral chemical potential. Right plot: variation of chiral susceptibility $\chi_{c}$ with temperature for finite magnetic field and finite chiral chemical potential. From this figure, it is clear that with increasing chiral chemical potential quark mass as well as the chiral transition temperature decreases. 
This decrease in mass with increasing chiral chemical potential has also manifested in the right plot of Fig. 6, which shows that with increasing chiral chemical potential chiral transition temperature decreases.

\section{CONCLUSION}

In this investigation, we have studied chiral phase transition and the associated chiral susceptibility of the medium produced in ultrarelativistic heavy ion collisions at vanishing quark chemical potential using Wigner function approach within the framework of two flavor NJL model. For a dynamical system, like the medium produced in heavy ion collision, quantum effects can be relevant. Hence, the quantum kinetic equation is a suitable formalism to understand the evolution of these dynamical system. The central quantity of the quantum kinetic description is the Wigner function. Wigner function is the quantum mechanical analog of classical distribution function. Different components of Wigner function satisfies quantum kinetic equation. However, in this investigation, we have restricted ourselves to the case of global equilibrium so that $T, \mu_{5}$ are constant and we do not consider evolution of Wigner function. In fact, we could have done the analysis by estimating the mean field thermodynamic potential and minimizing the same to get the quark masses as well as the susceptibility.

We have looked into the behavior of quark masses and chiral susceptibility within a two flavor NJL model with flavor mixing determinant interaction. In the absence of magnetic field, $u$ and $d$ quark masses are degenerate, due to the isospin symmetry. However, in the presence of magnetic field, due to different electric charge of $u$ and $d$ quark, constituent mass of $u$ and $d$ quark can be different. Our results show that while flavor mixing instanton induced interaction does not affect the quark masses in the absence of magnetic field; however, in the presence of magnetic field, this interaction can affect quark masses. For maximal flavor mixing, i.e., $\alpha=0.5$ in NJL model for a nonvanishing magnetic field, $u$ and $d$ quark masses are degenerate as the mass gap equation for $M_{u}$ and $M_{d}$ depend upon the sum of two condensates $\left(\left\langle\bar{\psi}_{u} \psi_{u}\right\rangle+\left\langle\bar{\psi}_{d} \psi_{d}\right\rangle\right)$. However, one has to keep in mind that this limiting case is not consistent with large $N_{c}$ limit of $G_{1}$ and $G_{2}$ as $G_{1} / G_{2} \sim N_{c}$ at large $N_{c}$. For nonmaximal flavor mixing, quark masses are nondegenerate in the presence of magnetic field. Constituent mass of $u$ and $d$ quark is larger for nonvanishing magnetic field compared to $B=0$ counterpart. With increasing magnetic field, constituent mass of $u$ and $d$ quark also increases. This apart the chiral transition temperature is higher for nonvanishing magnetic field as compared to the case of vanishing magnetic field. This is the manifestation of magnetic catalysis, i.e., in the presence of magnetic field the formation of chiral condensate is preferred even if the four Fermi coupling is below the critical coupling [25]. Further, the magnitude of the chiral condensate is higher for larger magnetic field. It is interesting to note that in the presence of nonmaximal flavor mixing instanton interaction, for vanishing magnetic field as well as for relatively small magnetic field, the chiral transition temperatures of $u$ and $d$ quark are the same. This is due to the fact that the mass difference between $u$ and $d$ quark arises due to the magnetic field and for weak magnetic field this difference is negligible and leads to the similar transition temperature. Only when this mass difference is large (due to strong enough magnetic field), one can have different transition temperature for two flavors. The difference between the transition temperature of $u$ and $d$ quark also increases with magnetic field. We have also shown that nonvanishing chiral chemical potential $\left(\mu_{5}\right)$ reduces quark mass in the absence as well as in the presence of magnetic field. Unlike magnetic catalysis, with increasing chiral chemical potential $\left(\mu_{5}\right)$, chiral transition temperature decreases. It is further observed that in the presence of magnetic field, the chiral susceptibility shows a double peak structure due to isospin breaking in the presence of magnetic field.

\section{ACKNOWLEDGMENTS}

We thank Aman Abhishek for useful discussions on MSS used in this work and also thank Jitesh R. Bhatt for useful discussions on Wigner function formalism. We would also like to thank M. Ruggieri and R. L. S. Farias for their valuable comments.

\section{APPENDIX A: DERIVATION OF SCALAR CONDENSATE IN A BACKGROUND MAGNETIC FIELD AND CHIRAL CHEMICAL POTENTIAL}

Scalar condensate in the terms of the scalar DHW function can be written as

$$
\langle\bar{\psi} \psi\rangle=\int d^{4} p F(p) .
$$

Using the explicit form of scalar DHW function $(F(p))$, as given in Eq. (43), scalar condensate in the presence of magnetic field, as given in Eq. (A1), can be expressed as 


$$
\begin{aligned}
\langle\bar{\psi} \psi\rangle= & \int 2 \pi p_{T} d p_{0} d p_{T} d p_{z} M\left[\sum_{n=0}^{\infty} V_{n}\left(p_{0}, p_{z}\right) \Lambda_{+}^{(n)}\left(p_{T}\right)+\sum_{n=1}^{\infty} \frac{1}{\sqrt{p_{z}^{2}+2 n q B}} A_{n}\left(p_{0}, p_{z}\right) p_{z} \Lambda_{-}^{(n)}\left(p_{T}\right)\right] \\
= & \int 2 \pi p_{T} d p_{0} d p_{T} d p_{z} M\left[V_{0}\left(p_{0}, p_{z}\right) \Lambda_{+}^{(0)}\left(p_{T}\right)+\sum_{n=1}^{\infty} V_{n}\left(p_{0}, p_{z}\right) \Lambda_{+}^{(n)}\left(p_{T}\right)\right. \\
& \left.+\sum_{n=1}^{\infty} \frac{1}{\sqrt{p_{z}^{2}+2 n q B}} A_{n}\left(p_{0}, p_{z}\right) p_{z} \Lambda_{-}^{(n)}\left(p_{T}\right)\right] \\
= & \mathbb{I}_{1}+\mathbb{I}_{2}+\mathbb{I}_{3} .
\end{aligned}
$$

Now, the first term in Eq. (A2),

$$
\mathbb{I}_{1}=2 \pi \iint d p_{0} d p_{z} M V_{0}\left(p_{0}, p_{z}\right) \int d p_{T} p_{T} \Lambda_{+}^{(0)}\left(p_{T}\right) .
$$

Using the explicit form of $V_{0}\left(p_{0}, p_{z}\right)$ and $\Lambda_{+}^{(0)}\left(p_{T}\right)$, Eq. (A3) can be expressed as

$$
\begin{aligned}
\mathbb{I}_{1}= & 2 \pi \iint d p_{0} d p_{z} \frac{2}{(2 \pi)^{3}} M \delta\left(\left(p_{0}+\mu\right)^{2}-\left|E_{p_{z}}^{(0)}\right|^{2}\right)\left[\theta\left(p_{0}+\mu\right) f_{F D}\left(p_{0}\right)\right. \\
& \left.+\theta\left(-p_{0}-\mu\right)\left[f_{F D}\left(-p_{0}\right)-1\right]\right] \int d p_{T} p_{T} 2 \exp \left[-p_{T}^{2} / q B\right] \\
= & \frac{q B}{(2 \pi)^{2}} \int d p_{z} \frac{M}{E_{p_{z}}^{(0)}}\left[f_{F D}\left(E_{p_{z}}^{(0)}-\mu\right)+f_{F D}\left(E_{p_{z}}^{(0)}+\mu\right)-1\right] .
\end{aligned}
$$

The second term in Eq. (A2),

$$
\mathbb{I}_{2}=2 \pi \sum_{n=1}^{\infty} \iint d p_{0} d p_{z} M V_{n}\left(p_{0}, p_{z}\right) \int d p_{T} p_{T} \Lambda_{+}^{(n)}\left(p_{T}\right)
$$

Using the explicit form of $\Lambda_{+}^{(n)}\left(p_{T}\right)$, one can calculate the following integral:

$$
\int d p_{T} p_{T} \Lambda_{+}^{(n)}\left(p_{T}\right)=(-1)^{n} \int_{0}^{\infty} d p_{T} p_{T}\left[L_{n}\left(2 p_{T}^{2} / q B\right)-L_{n-1}\left(2 p_{T}^{2} / q B\right)\right] \exp \left(-p_{T}^{2} / q B\right)=q B
$$

To get Eq. (A6), we use the following identity [114]:

$$
\int_{0}^{\infty} d x \exp (-b x) L_{n}(x)=(b-1)^{n} b^{-n-1} .
$$

Using Eq. (A6) and the explicit form of $V_{n}\left(p_{0}, p_{z}\right), \mathbb{I}_{2}$ can be written as

$$
\begin{aligned}
\mathbb{I}_{2} & =2 \pi(q B) \iint d p_{0} d p_{z} \frac{2}{(2 \pi)^{3}} M \sum_{s} \delta\left(\left(p_{0}+\mu\right)^{2}-\left|E_{p_{z}, s}^{(n)}\right|^{2}\right)\left[\theta\left(p_{0}+\mu\right) f_{F D}\left(p_{0}\right)+\theta\left(-p_{0}-\mu\right)\left(f_{F D}\left(-p_{0}\right)-1\right)\right] \\
& =-\frac{q B}{(2 \pi)^{2}} \sum_{n=1}^{\infty} \sum_{s} \int d p_{z} \frac{M}{E_{p z, s}^{(n)}}\left[1-f_{F D}\left(E_{p_{z}, s}^{(n)}-\mu\right)-f_{F D}\left(E_{p_{z}, s}^{(n)}+\mu\right)\right] .
\end{aligned}
$$

Now let us consider the third term of Eq. (A2),

$$
\mathbb{I}_{3}=2 \pi \iint d p_{0} d p_{z} M \sum_{n=1}^{\infty} \frac{1}{\sqrt{p_{z}^{2}+2 n q B}} A_{n}\left(p_{0}, p_{z}\right) p_{z} \int d p_{T} p_{T} \Lambda_{-}^{(n)}\left(p_{T}\right) .
$$


Using the explicit form of $\Lambda_{-}^{(n)}\left(p_{T}\right)$, it can be shown that

$$
\int d p_{T} p_{T} \Lambda_{-}^{(n)}\left(p_{T}\right)=0
$$

Hence, the third term of Eq. (A2),

$$
\mathbb{I}_{3}=0
$$

Hence, using Eqs. (A4), (A8), and (A11), the scalar condensate is

$$
\begin{aligned}
\langle\bar{\psi} \psi\rangle= & -\frac{q B}{(2 \pi)^{2}} \int d p_{z} \frac{M}{E_{p_{z}}^{(0)}}\left[1-f_{F D}\left(E_{p_{z}}^{(0)}-\mu\right)-f_{F D}\left(E_{p_{z}}^{(0)}+\mu\right)\right] \\
& -\frac{q B}{(2 \pi)^{2}} \sum_{n=1}^{\infty} \sum_{s} \int d p_{z} \frac{M}{E_{p_{z}, s}^{(n)}}\left[1-f_{F D}\left(E_{p_{z}, s}^{(n)}-\mu\right)-f_{F D}\left(E_{p_{z}, s}^{(n)}+\mu\right)\right] .
\end{aligned}
$$

\section{APPENDIX B: REGULARIZATION OF CHIRAL CONDENSATE IN A BACKGROUND MAGNETIC FIELD}

The scalar condensate of a quark of flavor $f$, with $N_{c}$ color d.o.f. at finite temperature $(T)$, chemical potential $(\mu)$ can be expressed as

$$
\begin{aligned}
\left\langle\bar{\psi}_{f} \psi_{f}\right\rangle_{B \neq 0}^{\mu_{5}=0} & =-\frac{N_{c}\left|q_{f}\right| B}{(2 \pi)^{2}} \sum_{n=0}^{\infty}\left(2-\delta_{n, 0}\right) \int d p_{z} \frac{M_{0_{f}}}{\epsilon_{p_{z}, f}^{(n)}}\left[1-f_{F D}\left(\epsilon_{p_{z}, f}^{(n)}-\mu\right)-f_{F D}\left(\epsilon_{p_{z}, f}^{(n)}+\mu\right)\right] \\
& =\left\langle\bar{\psi}_{f} \psi_{f}\right\rangle_{\text {vac, } \mathrm{B} \neq 0}^{\mu_{5}=0}+\left\langle\bar{\psi}_{f} \psi_{f}\right\rangle_{\text {med, } \mathrm{B} \neq 0}^{\mu_{5}=0},
\end{aligned}
$$

where $\left\langle\bar{\psi}_{f} \psi_{f}\right\rangle_{\text {vac,B } \mathrm{B} \neq 0}^{\mu_{5}=0}$ is the $T=0, \mu=0$ part, or the vacuum part of the scalar condensate, and $\left\langle\bar{\psi}_{f} \psi_{f}\right\rangle_{\text {med, } \mathrm{B} \neq 0}^{\mu_{5}=0}$ is the finite temperature and finite chemical potential part or the medium part of the scalar condensate in the presence of magnetic field. It is clear from Eq. (B1) the vacuum term is divergent for large momenta and however because of the distribution functions the medium part in Eq. (B1) is not. Hence, it is important to regulate the vacuum part in Eq. (B1).

Let us consider the vacuum part $\left\langle\bar{\psi}_{f} \psi_{f}\right\rangle_{\text {vac }, \mathrm{B} \neq 0}^{\mu_{5}=0}$, which is given as

$$
\begin{aligned}
\left\langle\bar{\psi}_{f} \psi_{f}\right\rangle_{\text {vac }, \mathrm{B} \neq 0}^{\mu_{5}=0} & =-\frac{N_{c}}{2 \pi} \sum_{n=0}^{\infty}\left(2-\delta_{n, 0}\right)\left|q_{f}\right| B \int_{-\infty}^{\infty} \frac{d p_{z}}{(2 \pi)} \frac{M_{0_{f}}}{\epsilon_{p_{z}, f}^{(n)}} \\
& =-\frac{N_{c}}{2 \pi} \sum_{n=0}^{\infty} 2\left|q_{f}\right| B \int_{-\infty}^{\infty} \frac{d p_{z}}{(2 \pi)} \frac{M_{0_{f}}}{\epsilon_{p_{z}, f}^{(n)}}+\frac{N_{c}}{2 \pi}\left|q_{f}\right| B \int_{-\infty}^{\infty} \frac{d p_{z}}{(2 \pi)} \frac{M_{0_{f}}}{\epsilon_{p_{z}, f}^{(0)}} \\
& =\mathcal{I}_{1}+\mathcal{I}_{2} .
\end{aligned}
$$

Both integrals $\mathcal{I}_{1}$ and $\mathcal{I}_{2}$ are divergent at large momentum. These integrals can be regularized using dimensional regularization scheme. In this regularization scheme, integral $\mathcal{I}_{1}$ can be expressed as

$$
\begin{aligned}
\mathcal{I}_{1} & =-\frac{N_{c}}{2 \pi} \sum_{n=0}^{\infty} 2\left|q_{f}\right| B \int_{-\infty}^{\infty} \frac{d p_{z}}{(2 \pi)} \frac{M_{0_{f}}}{\epsilon_{p_{z}, f}^{(n)}} \\
& =-\frac{N_{c}}{2 \pi} \sum_{n=0}^{\infty} 2\left|q_{f}\right| B \frac{M_{0_{f}} \Gamma(\epsilon / 2)}{(4 \pi)^{(1-\epsilon) / 2} \Gamma(1 / 2)\left(x_{0_{f}}+n\right)^{\epsilon / 2}},
\end{aligned}
$$

where the dimensionless variable $x_{0_{f}} \equiv M_{0_{f}}^{2} / 2\left|q_{f}\right| B$. Similarly, the integral $\mathcal{I}_{2}$ can be expressed as 


$$
\begin{aligned}
\mathcal{I}_{2} & =\frac{N_{c}}{2 \pi}\left|q_{f}\right| B \int \frac{d p_{z}}{(2 \pi)} \frac{M_{0_{f}}}{\sqrt{M_{0_{f}}^{2}+p_{z}^{2}}} \\
& =\frac{N_{c} M_{0_{f}}\left|q_{f}\right| B}{(2 \pi)} \frac{\Gamma(\epsilon / 2)}{(4 \pi)^{(1-\epsilon) / 2} \Gamma(1 / 2) x_{0_{f}}^{\epsilon / 2}} .
\end{aligned}
$$

Using Eqs. (B3) and (B4), vacuum part of the scalar condensate in the presence of magnetic field as given in Eq. (B2) can be recasted as

$$
\begin{aligned}
\mathcal{I}_{1}+\mathcal{I}_{2} & =-\frac{N_{c}}{2 \pi} 2\left|q_{f}\right| B M_{0_{f}} \frac{\Gamma(\epsilon / 2)}{(4 \pi)^{(1-\epsilon) / 2} \Gamma(1 / 2)}\left[\sum_{n=0}^{\infty} \frac{1}{\left(x_{0_{f}}+n\right)^{\epsilon / 2}}-\frac{1}{2 x_{0_{f}}^{\epsilon / 2}}\right] \\
& =-\frac{N_{c}}{2 \pi} 2\left|q_{f}\right| B M_{0_{f}} \frac{\Gamma(\epsilon / 2)}{(4 \pi)^{1 / 2} \Gamma(1 / 2)}\left[\zeta\left(\epsilon / 2, x_{0_{f}}\right)-\frac{1}{2 x_{0_{f}}^{\epsilon / 2}}\right] .
\end{aligned}
$$

Expanding the right-hand side of Eq. (B5) around $\epsilon \rightarrow 0$ and keeping only the leading order terms, we get

$$
\mathcal{I}_{1}+\mathcal{I}_{2}=-\frac{N_{c}}{2 \pi^{2}}\left|q_{f}\right| B M_{0_{f}}\left[-\frac{2 x_{0_{f}}}{\epsilon}+\gamma_{E} x_{0_{f}}+\frac{1}{2} \ln x_{0_{f}}+\ln \Gamma\left(x_{0_{f}}\right)-\frac{1}{2} \ln (2 \pi)\right] \text {. }
$$

In Eq. (B5), we have used the representation of zeta function, which is given as [115]

$$
\zeta(a, x)=\sum_{n=0}^{\infty} \frac{1}{(x+n)^{a}} .
$$

Also, we have used the following identities to get Eq. (B6):

$$
\zeta(0, x)=\left(\frac{1}{2}-x\right), \quad \text { and }, \quad \zeta^{\prime}(0, x)=\ln \Gamma(x)-\frac{1}{2} \ln (2 \pi), \quad \text { where } \zeta^{\prime}(0, x)=\left.\frac{d \zeta(a, x)}{d a}\right|_{a=0} .
$$

It is clear from Eq. (B6) that the vacuum part has $1 / \epsilon$ divergent part. To remove this $1 / \epsilon$ divergence, we use the following integral:

$$
\mathcal{I}_{3}=-2 N_{c} \int \frac{d^{3} p}{(2 \pi)^{3}} \frac{M_{0_{f}}}{\sqrt{p^{2}+M_{0_{f}}^{2}}} .
$$

Using dimensional regularization method, the integral in Eq. (B9) can be recasted as

$$
\mathcal{I}_{3}=\frac{-2 N_{c} M_{0_{f}}}{(4 \pi)^{3 / 2} \Gamma(1 / 2)} \frac{\Gamma(-1+\epsilon / 2)}{\left(2 x_{0_{f}}\left|q_{f}\right| B\right)^{-1+\epsilon / 2}} .
$$

Expand the right-hand side of Eq. (B10) around $\epsilon \rightarrow 0$ and keeping only the leading order terms, we get

$$
\mathcal{I}_{3}=\frac{-N_{c} M_{0_{f}}\left|q_{f}\right| B}{2 \pi^{2}}\left[-\frac{2 x_{0_{f}}}{\epsilon}-x_{0_{f}}+x_{0_{f}} \gamma_{E}+x_{0_{f}} \ln x_{0_{f}}\right] .
$$

Using Eqs. (B6) and (B11), we get

$$
\mathcal{I}_{1}+\mathcal{I}_{2}-\mathcal{I}_{3}=-\frac{N_{c} M_{0_{f}}\left|q_{f}\right| B}{2 \pi^{2}}\left[x_{0_{f}}\left(1-\ln x_{0_{f}}\right)+\ln \Gamma\left(x_{0_{f}}\right)+\frac{1}{2} \ln \left(\frac{x_{0_{f}}}{2 \pi}\right)\right] .
$$


Using Eqs. (B2) and (B12), we have the regularized vacuum part of the scalar condensate in the presence of magnetic field and is given as

$$
\begin{aligned}
\left\langle\bar{\psi}_{f} \psi_{f}\right\rangle_{\text {vac, } \mathrm{B} \neq 0}^{\mu_{5}=0} & =\mathcal{I}_{1}+\mathcal{I}_{2}-\mathcal{I}_{3}+\mathcal{I}_{3} \\
& =-\frac{N_{c} M_{0_{f}}\left|q_{f}\right| B}{2 \pi^{2}}\left[x_{0_{f}}\left(1-\ln x_{0_{f}}\right)+\ln \Gamma\left(x_{0_{f}}\right)+\frac{1}{2} \ln \left(\frac{x_{0_{f}}}{2 \pi}\right)\right]-2 N_{c} \int_{|\vec{p}| \leq \Lambda} \frac{d^{3} p}{(2 \pi)^{3}} \frac{M_{0_{f}}}{\sqrt{p^{2}}+M_{0_{f}}^{2}}
\end{aligned}
$$

Again,

$$
\begin{aligned}
\mathcal{I}_{1} & =\mathcal{I}_{1}-\mathcal{I}_{3}+\mathcal{I}_{3} \\
& =-\frac{N_{c}\left|q_{f}\right| B M_{0_{f}}}{2 \pi^{2}}\left[\frac{1}{\epsilon}-\frac{\gamma_{E}}{2}+x_{0_{f}}\left(1-\ln x_{0_{f}}\right)+\ln \Gamma\left(x_{0_{f}}\right)-\frac{1}{2} \ln (2 \pi)\right]-2 N_{c} \int \frac{d^{3} p}{(2 \pi)^{3}} \frac{M_{0_{f}}}{\sqrt{p^{2}+M_{0_{f}}^{2}}}
\end{aligned}
$$

Hence,

$$
\begin{aligned}
\mathcal{I} & \equiv-\frac{N_{c}\left|q_{f}\right| B}{(2 \pi)^{2}} \sum_{s= \pm 1} \sum_{n=0}^{\infty} \int d p_{z} \frac{1}{\sqrt{p_{z}^{2}+M_{0_{f}}^{2}+2 n\left|q_{f}\right| B}} \\
& =-\frac{N_{c}}{2 \pi^{2}}\left|q_{f}\right| B\left[\frac{1}{\epsilon}-\frac{\gamma_{E}}{2}+x_{0_{f}}\left(1-\ln x_{0_{f}}\right)+\ln \Gamma\left(x_{0_{f}}\right)-\frac{1}{2} \ln (2 \pi)\right]-2 N_{c} \int \frac{d^{3} p}{(2 \pi)^{3}} \frac{1}{\sqrt{p^{2}+M_{0_{f}}^{2}}} .
\end{aligned}
$$

Using Eq. (B15), we get

$$
\begin{aligned}
\frac{N_{c}\left|q_{f}\right| B}{(2 \pi)^{2}} \sum_{s= \pm 1} \sum_{n=0}^{\infty} \int d p_{z} \frac{1}{\left(p_{z}^{2}+M_{0_{f}}^{2}+2 n\left|q_{f}\right| B\right)^{3 / 2}} & \equiv \frac{1}{M_{0_{f}}} \frac{\partial \mathcal{I}}{\partial M_{0_{f}}} \\
& =-\frac{N_{c}}{2 \pi^{2}}\left[-\ln x_{0_{f}}+\frac{\Gamma^{\prime}\left(x_{0_{f}}\right)}{\Gamma\left(x_{0_{f}}\right)}\right]+2 N_{c} \int_{|\vec{p}| \leq \Lambda} \frac{d^{3} p}{(2 \pi)^{3}} \frac{1}{\left(p^{2}+M_{0_{f}}^{2}\right)^{3 / 2}} .
\end{aligned}
$$

\section{APPENDIX C: REGULARIZATION OF CHIRAL CONDENSATE IN A BACKGROUND MAGNETIC FIELD AND CHIRAL CHEMICAL POTENTIAL}

The scalar condensate of a quark of flavor $f$ with $N_{c}$ color d.o.f. at finite temperature $(T)$, quark chemical potential $(\mu)$, chiral chemical potential $\left(\mu_{5}\right)$, electric charge $\left(q_{f}\right)$, and magnetic field $(B)$ can be expressed as

$$
\begin{aligned}
\left\langle\bar{\psi}_{f} \psi_{f}\right\rangle_{B \neq 0}^{\mu_{5} \neq 0}= & -\frac{N_{c}\left|q_{f}\right| B}{(2 \pi)^{2}}\left[\int d p_{z} \frac{M_{f}}{E_{p_{z}, f}^{(0)}}\left[1-f_{F D}\left(E_{p_{z}, f}^{(0)}-\mu\right)-f_{F D}\left(E_{p_{z}, f}^{(0)}+\mu\right)\right]\right. \\
& \left.+\sum_{n=1}^{\infty} \sum_{s} \int d p_{z} \frac{M_{f}}{E_{p_{z}, s, f}^{(n)}}\left[1-f_{F D}\left(E_{p_{z}, s, f}^{(n)}-\mu\right)-f_{F D}\left(E_{p_{z}, s, f}^{(n)}+\mu\right)\right]\right] \\
= & \left\langle\bar{\psi}_{f} \psi_{f}\right\rangle_{\substack{\text { vac }, \mathrm{B} \neq 0 \\
\mu_{1} \neq 0}}+\left\langle\bar{\psi}_{f} \psi_{f}\right\rangle_{\text {med,B } \neq 0}^{\mu_{5} \neq 0},
\end{aligned}
$$

where $\left\langle\bar{\psi}_{f} \psi_{f}\right\rangle_{\text {vac,B } \neq 0}^{\mu_{5} \neq 0}$ is the $T=0, \mu=0$ part or the vacuum part of the scalar condensate, and $\left\langle\bar{\psi}_{f} \psi_{f}\right\rangle_{\text {med,B } \neq 0}^{\mu_{5} \neq 0}$ is the finite temperature and finite chemical potential part or the medium part of the scalar condensate in the presence of magnetic field and chiral chemical potential $\left(\mu_{5}\right)$. It is clear from Eq. (C1) that the vacuum term is divergent at large momenta and however because of the distribution functions the medium part in Eq. (C1) is not. Hence, the vacuum term has to be regularized. 
The vacuum term in the presence of magnetic field and chiral chemical potential can be expressed as

$$
\begin{aligned}
\left\langle\bar{\psi}_{f} \psi_{f}\right\rangle_{\mathrm{vac}, \mathrm{B} \neq 0}^{\mu_{5} \neq 0}= & -N_{c} \frac{\left|q_{f}\right| B}{(2 \pi)^{2}} \int d p_{z} \frac{M_{f}}{\sqrt{M_{f}^{2}+\left(p_{z}-\mu_{5}\right)^{2}}}-N_{c} \frac{\left|q_{f}\right| B}{(2 \pi)^{2}} \sum_{n=1}^{\infty} \sum_{s= \pm 1} \int d p_{z} \frac{M_{f}}{\sqrt{M_{f}^{2}+\left(\sqrt{p_{z}^{2}+2 n\left|q_{f}\right| B}-s \mu_{5}\right)^{2}}}+N_{c} \frac{\left|q_{f}\right| B}{(2 \pi)^{2}} \int d p_{z} \frac{M_{f}}{\sqrt{M_{f}^{2}+\left(p_{z}-\mu_{5}\right)^{2}}} \\
= & -N_{c} \frac{\left|q_{f}\right| B}{(2 \pi)^{2}} \sum_{n=0}^{\infty} \sum_{s= \pm 1} \int d p_{z} \frac{M_{f}}{\sqrt{M_{f}^{2}+\left(\sqrt{p_{z}^{2}+2 n\left|q_{f}\right| B}-s \mu_{5}\right)^{2}}} \\
= & -N_{c} \frac{\left|q_{f}\right| B}{(2 \pi)^{2}} \sum_{n=0}^{\infty} \sum_{s= \pm 1} \int d p_{z} \frac{1}{\pi} \int_{-\infty}^{\infty} d p_{4} \frac{M_{f}}{p_{4}^{2}+\left(M_{f}^{2}+\left(\sqrt{p_{z}^{2}+2 n\left|q_{f}\right| B}-s \mu_{5}\right)^{2}\right)} \\
& +N_{c} \frac{\left|q_{f}\right| B}{(2 \pi)^{2}} \int d p_{z} \frac{1}{\pi} \int_{-\infty}^{\infty} d p_{4} \frac{M_{f}}{p_{4}^{2}+M_{f}^{2}+\left(p_{z}-\mu_{5}\right)^{2}} \\
= & I_{1}+I_{2} .
\end{aligned}
$$

Using the regularization method discussed in Ref. [59], we can write the integrand of the integral $I_{1}$ as given in the Eq. (C2) as follows:

$$
\begin{aligned}
& \frac{1}{p_{4}^{2}+M_{f}^{2}+\left(\sqrt{p_{z}^{2}+2 n\left|q_{f}\right| B}-s \mu_{5}\right)^{2}} \\
& =\frac{1}{p_{4}^{2}+p_{z}^{2}+M_{0_{f}}^{2}+2 n\left|q_{f}\right| B}-\frac{1}{p_{4}^{2}+p_{z}^{2}+M_{0_{f}}^{2}+2 n\left|q_{f}\right| B}+\frac{1}{p_{4}^{2}+M_{f}^{2}+\left(\sqrt{p_{z}^{2}+2 n\left|q_{f}\right| B}-s \mu_{5}\right)^{2}} \\
& =\frac{1}{p_{4}^{2}+p_{z}^{2}+M_{0_{f}}^{2}+2 n\left|q_{f}\right| B}+\frac{M_{0_{f}}^{2}-M_{f}^{2}-\mu_{5}^{2}+2 s \mu_{5} \sqrt{p_{z}^{2}+2 n\left|q_{f}\right| B}}{\left(p_{4}^{2}+p_{z}^{2}+M_{0_{f}}^{2}+2 n\left|q_{f}\right| B\right)\left(p_{4}^{2}+M_{f}^{2}+\left(\sqrt{p_{z}^{2}+2 n\left|q_{f}\right| B}-s \mu_{5}\right)^{2}\right)} .
\end{aligned}
$$

Using Eq. (C3) twice, we can write the integrand of the integral $I_{1}$ in the following way:

$$
\begin{aligned}
\frac{1}{p_{4}^{2}+M_{f}^{2}+\left(\sqrt{p_{z}^{2}+2 n\left|q_{f}\right| B}-s \mu_{5}\right)^{2}}= & \frac{1}{p_{4}^{2}+p_{z}^{2}+M_{0_{f}}^{2}+2 n\left|q_{f}\right| B}+\frac{A+2 s \mu_{5} \sqrt{p_{z}^{2}+2 n\left|q_{f}\right| B}}{\left(p_{4}^{2}+p_{z}^{2}+M_{0_{f}}^{2}+2 n\left|q_{f}\right| B\right)^{2}} \\
& +\frac{\left(A+2 s \mu_{5} \sqrt{p_{z}^{2}+2 n\left|q_{f}\right| B}\right)^{2}}{\left(p_{4}^{2}+p_{z}^{2}+M_{0_{f}}^{2}+2 n\left|q_{f}\right| B\right)^{3}} \\
& +\frac{\left(A+2 s \mu_{5} \sqrt{p_{z}^{2}+2 n\left|q_{f}\right| B}\right)^{3}}{\left(p_{4}^{2}+p_{z}^{2}+M_{0_{f}}^{2}+2 n\left|q_{f}\right| B\right)^{3}\left(p_{4}^{2}+M_{f}^{2}+\left(\sqrt{p_{z}^{2}+2 n\left|q_{f}\right| B}-s \mu_{5}\right)^{2}\right)},
\end{aligned}
$$

where $A=M_{0_{f}}^{2}-M_{f}^{2}-\mu_{5}^{2}$. Performing $p_{4}$ integration in each term of Eq. (C4), we get

$$
\frac{1}{\pi} \sum_{s} \int d p_{4} \frac{1}{p_{4}^{2}+p_{z}^{2}+M_{0_{f}}^{2}+2 n\left|q_{f}\right| B}=\sum_{s} \frac{1}{\sqrt{p_{z}^{2}+M_{0_{f}}^{2}+2 n\left|q_{f}\right| B}}
$$




$$
\begin{aligned}
& \frac{1}{\pi} \sum_{s} \int d p_{4} \frac{A+2 s \mu_{5} \sqrt{p_{z}^{2}+2 n\left|q_{f}\right| B}}{\left(p_{4}^{2}+p_{z}^{2}+M_{0_{f}}^{2}+2 n\left|q_{f}\right| B\right)^{2}}=\sum_{s} \frac{1}{2} \frac{A}{\left(p_{z}^{2}+M_{0_{f}}^{2}+2 n\left|q_{f}\right| B\right)^{3 / 2}} \\
& \frac{1}{\pi} \sum_{s} \int d p_{4} \frac{\left(A+2 s \mu_{5} \sqrt{p_{z}^{2}+2 n\left|q_{f}\right| B}\right)^{2}}{\left(p_{4}^{2}+p_{z}^{2}+M_{0_{f}}^{2}+2 n\left|q_{f}\right| B\right)^{3}}=\sum_{s}\left[\frac{3}{8} \frac{A^{2}}{\left(p_{z}^{2}+M_{0_{f}}^{2}+2 n\left|q_{f}\right| B\right)^{5 / 2}}-\frac{3}{2} \frac{\mu_{5}^{2} M_{0_{f}}^{2}}{\left(p_{z}^{2}+M_{0_{f}}^{2}+2 n\left|q_{f}\right| B\right)^{5 / 2}}\right. \\
& \left.+\frac{3}{2} \frac{\mu_{5}^{2}}{\left(p_{z}^{2}+M_{0_{f}}^{2}+2 n\left|q_{f}\right| B\right)^{3 / 2}}\right] \\
& \frac{1}{\pi} \sum_{s} \int d p_{4} \frac{\left(A+2 s \mu_{5} \sqrt{p_{z}^{2}+2 n\left|q_{f}\right| B}\right)^{3}}{\left(p_{4}^{2}+p_{z}^{2}+M_{0_{f}}^{2}+2 n\left|q_{f}\right| B\right)^{3}\left(p_{4}^{2}+M_{f}^{2}+\left(\sqrt{p_{z}^{2}+2 n\left|q_{f}\right| B}-s \mu_{5}\right)^{2}\right)} \\
& =\frac{1}{\pi} \sum_{s} \int d p_{4} \int_{0}^{1} d x \frac{3(1-x)^{2}\left(A+2 s \mu_{5} \sqrt{p_{z}^{2}+2 n\left|q_{f}\right| B}\right)^{3}}{\left[x\left(p_{4}^{2}+M_{f}^{2}+\left(\sqrt{p_{z}^{2}+2 n\left|q_{f}\right| B}-s \mu_{5}\right)^{2}\right)+(1-x)\left(p_{4}^{2}+p_{z}^{2}+M_{0_{f}}^{2}+2 n\left|q_{f}\right| B\right)\right]^{4}} \\
& =\sum_{s} \frac{15}{16} \int_{0}^{1} d x \frac{(1-x)^{2}\left(A+2 s \mu_{5} \sqrt{p_{z}^{2}+2 n\left|q_{f}\right| B}\right)^{3}}{\left[p_{z}^{2}+M_{0_{f}}^{2}+2 n\left|q_{f}\right| B-x\left(A+2 s \mu_{5} \sqrt{p_{z}^{2}+2 n\left|q_{f}\right| B}\right)\right]^{7 / 2}} .
\end{aligned}
$$

Using Eqs. (C5)-(C8), integral $I_{1}$ in Eq. (C2) can be expressed as

$$
\begin{aligned}
I_{1} & =-N_{c} \frac{\left|q_{f}\right| B}{(2 \pi)^{2}} \sum_{n=0}^{\infty} \sum_{s= \pm 1} \int d p_{z} \frac{M_{f}}{\sqrt{M_{f}^{2}+\left(\sqrt{p_{z}^{2}+2 n\left|q_{f}\right| B}-s \mu_{5}\right)^{2}}} \\
& =I_{1_{\text {quad }}}-\frac{M_{f}\left(M_{0_{f}}^{2}-M_{f}^{2}+2 \mu_{5}^{2}\right)}{2} I_{1_{\text {log }}}+I_{1_{\text {finitel }}}+I_{1_{\text {finite } 2}},
\end{aligned}
$$

where

$$
\begin{gathered}
I_{1_{\text {quad }}}=-N_{c} \frac{\left|q_{f}\right| B}{(2 \pi)^{2}} \sum_{n=0}^{\infty} \sum_{s= \pm 1} \int d p_{z} \frac{M_{f}}{\sqrt{p_{z}^{2}+M_{0_{f}}^{2}+2 n\left|q_{f}\right| B}}, \\
I_{1_{\log }}=N_{c} \frac{\left|q_{f}\right| B}{(2 \pi)^{2}} \sum_{n=0}^{\infty} \sum_{s= \pm 1} \int d p_{z} \frac{1}{\left(p_{z}^{2}+M_{0_{f}}^{2}+2 n\left|q_{f}\right| B\right)^{3 / 2}}, \\
I_{1_{\text {finitel }}}=-N_{c} \frac{\left|q_{f}\right| B}{(2 \pi)^{2}} \sum_{n=0}^{\infty} \sum_{s= \pm 1} \int d p_{z}\left(\frac{3}{8}\right)\left[\frac{M_{f} A^{2}-4 M_{f} M_{0_{f}}^{2} \mu_{5}^{2}}{\left(p_{z}^{2}+M_{0_{f}}^{2}+2 n\left|q_{f}\right| B\right)^{5 / 2}}\right] \\
I_{1_{\text {finite }}}=-N_{c} \frac{\left|q_{f}\right| B}{(2 \pi)^{2}}\left(\frac{15}{16}\right) \sum_{n=0}^{\infty} \sum_{s= \pm 1} \int d p_{z} \int_{0}^{1} d x \frac{(1-x)^{2} M_{f}\left(A+2 s \mu_{5} \sqrt{p_{z}^{2}+2 n\left|q_{f}\right| B}\right)^{3}}{\left[p_{z}^{2}+M_{0_{f}}^{2}+2 n\left|q_{f}\right| B-x\left(A+2 s \mu_{5} \sqrt{p_{z}^{2}+2 n\left|q_{f}\right| B}\right)\right]^{7 / 2}} .
\end{gathered}
$$


In a similar way, the integral $I_{2}$ in Eq. (C2) can also be written as

$$
\begin{aligned}
I_{2} & =N_{c} \frac{\left|q_{f}\right| B}{(2 \pi)^{2}} \int d p_{z} \frac{M_{f}}{\sqrt{M_{f}^{2}+\left(p_{z}-\mu_{5}\right)^{2}}} \\
& =N_{c} \frac{\left|q_{f}\right| B}{(2 \pi)^{2}} \int d p_{z} \frac{M_{f}}{\sqrt{M_{f}^{2}+\left(p_{z}-\mu_{5}\right)^{2}}}-N_{c} \frac{\left|q_{f}\right| B}{(2 \pi)^{2}} \int d p_{z} \frac{M_{f}}{\sqrt{p_{z}^{2}+M_{0_{f}}^{2}}}+N_{c} \frac{\left|q_{f}\right| B}{(2 \pi)^{2}} \int d p_{z} \frac{M_{f}}{\sqrt{p_{z}^{2}+M_{0_{f}}^{2}}} \\
& =\left(\frac{1}{2}\right) N_{c} \frac{\left|q_{f}\right| B}{(2 \pi)^{2}} \int d p_{z} \int_{0}^{1} d x \frac{M_{f}\left(A+2 p_{z} \mu_{5}\right)}{\left[p_{z}^{2}+M_{0_{f}}^{2}-x\left(A+2 p_{z} \mu_{5}\right)\right]^{3 / 2}}+N_{c} \frac{\left|q_{f}\right| B}{(2 \pi)^{2}} \int d p_{z} \frac{M_{f}}{\sqrt{p_{z}^{2}+M_{0_{f}}^{2}}} \\
& =I_{2_{\text {finite }}}+I_{2_{\log }} .
\end{aligned}
$$

Using Eqs. (C9) and (C14), Eq. (C2) can be recasted as

$$
\left\langle\bar{\psi}_{f} \psi_{f}\right\rangle_{\mathrm{vac}, \mathrm{B} \neq 0}^{\mu_{5} \neq 0}=-\frac{M_{f}\left(M_{0_{f}}^{2}-M_{f}^{2}+2 \mu_{5}^{2}\right)}{2} I_{1_{\log }}+I_{1_{\text {finitel }}}+I_{1_{\text {finite } 2}}+I_{2_{\text {finite }}}+I_{1_{\text {quad }}}+I_{2_{\log }},
$$

where

$$
I_{1_{\log }}=-\frac{N_{c}}{2 \pi^{2}}\left[-\ln x_{0_{f}}+\frac{\Gamma^{\prime}\left(x_{0_{f}}\right)}{\Gamma\left(x_{0_{f}}\right)}\right]+2 N_{c} \int_{|\vec{p}| \leq \Lambda} \frac{d^{3} p}{(2 \pi)^{3}} \frac{1}{\left(p^{2}+M_{0_{f}}^{2}\right)^{3 / 2}},
$$

and

$$
I_{1_{\text {quad }}}+I_{2_{\log }}=-\frac{N_{c} M_{f}\left|q_{f}\right| B}{2 \pi^{2}}\left[x_{0_{f}}\left(1-\ln x_{0_{f}}\right)+\ln \Gamma\left(x_{0_{f}}\right)+\frac{1}{2} \ln \left(\frac{x_{0_{f}}}{2 \pi}\right)\right]-2 N_{c} \int_{|\vec{p}| \leq \Lambda} \frac{d^{3} p}{(2 \pi)^{3}} \frac{M_{f}}{\sqrt{p^{2}+M_{0_{f}}^{2}}} .
$$

In Eqs. (C16) and (C17), we have used Eqs. (B16) and (B13), respectively.

\section{APPENDIX D: CHIRAL SUSCEPTIBILITY AND ITS REGULARIZATION IN THE PRESENCE OF A BACKGROUND MAGNETIC FIELD AND CHIRAL CHEMICAL POTENTIAL}

Using Eq. (C2), we get

$$
\begin{aligned}
\frac{\partial\left\langle\bar{\psi}_{f} \psi_{f}\right\rangle_{\mathrm{vac}, \mathrm{B} \neq 0}^{\mu_{5} \neq 0}}{\partial M_{f}}= & -\frac{N_{c}\left|q_{f}\right| B}{(2 \pi)^{2}} \sum_{n=0}^{\infty} \sum_{s= \pm 1} \int d p_{z} \frac{1}{\sqrt{M_{f}^{2}+\left(\sqrt{p_{z}^{2}+2 n\left|q_{f}\right| B}-s \mu_{5}\right)^{2}}} \\
& +\frac{N_{c}\left|q_{f}\right| B}{(2 \pi)^{2}} \int d p_{z} \frac{1}{\sqrt{M_{f}^{2}+\left(p_{z}-\mu_{5}\right)^{2}}} \\
& +\frac{N_{c}\left|q_{f}\right| B}{(2 \pi)^{2}} \sum_{n=0}^{\infty} \sum_{s= \pm 1} \int d p_{z} \frac{M_{f}^{2}}{\left(M_{f}^{2}+\left(\sqrt{p_{z}^{2}+2 n\left|q_{f}\right| B}-s \mu_{5}\right)^{2}\right)^{3 / 2}} \\
& -\frac{N_{c}\left|q_{f}\right| B}{(2 \pi)^{2}} \int d p_{z} \frac{M_{f}^{2}}{\left(M_{f}^{2}+\left(p_{z}-\mu_{5}\right)^{2}\right)^{3 / 2}} \\
= & \mathbf{I}_{1}+\mathbf{I}_{2}+\mathbf{I}_{3}+\mathbf{I}_{4} .
\end{aligned}
$$


Using Eq. (C9), we can write

$$
\begin{aligned}
\mathbf{I}_{1} & =-N_{c} \frac{\left|q_{f}\right| B}{(2 \pi)^{2}} \sum_{n=0}^{\infty} \sum_{s= \pm 1} \int d p_{z} \frac{1}{\sqrt{M_{f}^{2}+\left(\sqrt{p_{z}^{2}+2 n\left|q_{f}\right| B}-s \mu_{5}\right)^{2}}} \\
& =\mathbf{I}_{1, \text { quad }}-\frac{\left(M_{0_{f}}^{2}-M_{f}^{2}+2 \mu_{5}^{2}\right)}{2} \mathbf{I}_{1, \text { log }}+\mathbf{I}_{1, \text { finite } 1}+\mathbf{I}_{1, \text { finite } 2},
\end{aligned}
$$

where

$$
\begin{gathered}
\mathbf{I}_{1, \text { quad }}=-N_{c} \frac{\left|q_{f}\right| B}{(2 \pi)^{2}} \sum_{n=0}^{\infty} \sum_{s= \pm 1} \int d p_{z} \frac{1}{\sqrt{p_{z}^{2}+M_{0_{f}}^{2}+2 n\left|q_{f}\right| B}}, \\
\mathbf{I}_{1, \text { log }}=N_{c} \frac{\left|q_{f}\right| B}{(2 \pi)^{2}} \sum_{n=0}^{\infty} \sum_{s= \pm 1} \int d p_{z} \frac{1}{\left(p_{z}^{2}+M_{0_{f}}^{2}+2 n\left|q_{f}\right| B\right)^{3 / 2}}, \\
\mathbf{I}_{1, \text { finite } 1}=-N_{c} \frac{\left|q_{f}\right| B}{(2 \pi)^{2}} \sum_{n=0}^{\infty} \sum_{s= \pm 1} \int d p_{z}\left(\frac{3}{8}\right)\left[\frac{A^{2}-4 M_{0_{f}}^{2} \mu_{5}^{2}}{\left(p_{z}^{2}+M_{0_{f}}^{2}+2 n\left|q_{f}\right| B\right)^{5 / 2}}\right] \\
\mathbf{I}_{1, \text { finite } 2}=-N_{c} \frac{\left|q_{f}\right| B}{(2 \pi)^{2}}\left(\frac{15}{16}\right) \sum_{n=0}^{\infty} \sum_{s= \pm 1} \int d p_{z} \int_{0}^{1} d x \frac{(1-x)^{2}\left(A+2 s \mu_{5} \sqrt{p_{z}^{2}+2 n\left|q_{f}\right| B}\right)^{3}}{\left[p_{z}^{2}+M_{0_{f}}^{2}+2 n\left|q_{f}\right| B-x\left(A+2 s \mu_{5} \sqrt{p_{z}^{2}+2 n\left|q_{f}\right| B}\right)\right]^{7 / 2}} .
\end{gathered}
$$

The integral $\mathbf{I}_{2}$ in Eq. (D1) can be expressed as

$$
\mathbf{I}_{2}=N_{c} \frac{\left|q_{f}\right| B}{(2 \pi)^{2}} \int d p_{z} \frac{1}{\sqrt{M_{f}^{2}+\left(p_{z}-\mu_{5}\right)^{2}}}=\mathbf{I}_{2, \text { finite }}+\mathbf{I}_{2, \log },
$$

where divergence free $\mathbf{I}_{2 \text {,finite }}$ is

$$
\mathbf{I}_{2, \text { finite }}=\left(\frac{1}{2}\right) N_{c} \frac{\left|q_{f}\right| B}{(2 \pi)^{2}} \int d p_{z} \int_{0}^{1} d x \frac{\left(A+2 p_{z} \mu_{5}\right)}{\left[p_{z}^{2}+M_{0_{f}}^{2}-x\left(A+2 p_{z} \mu_{5}\right)\right]^{3 / 2}},
$$

and the divergence term $\mathbf{I}_{2, \log }$ is

$$
\mathbf{I}_{2, \log }=\frac{N_{c}\left|q_{f}\right| B}{(2 \pi)^{2}} \int d p_{z} \frac{1}{\sqrt{p_{z}^{2}+M_{0_{f}}^{2}}} .
$$

Similarly, the integral $\mathbf{I}_{3}$ can be separated into a divergent term and a convergent term as

$$
\mathbf{I}_{3}=\frac{N_{c}\left|q_{f}\right| B}{(2 \pi)^{2}} \sum_{n=0}^{\infty} \sum_{s= \pm 1} \int d p_{z} \frac{M_{f}^{2}}{\left(M_{f}^{2}+\left(\sqrt{p_{z}^{2}+2 n\left|q_{f}\right| B}-s \mu_{5}\right)^{2}\right)^{3 / 2}}=\mathbf{I}_{3, \text { finite }}+\mathbf{I}_{3, \log },
$$

where

$$
\mathbf{I}_{3, \text { finite }}=\frac{N_{c}\left|q_{f}\right| B}{(2 \pi)^{2}} \sum_{n=0}^{\infty} \sum_{s= \pm 1} \int d p_{z} M_{f}^{2}\left[\frac{1}{\left(M_{f}^{2}+\left(\sqrt{p_{z}^{2}+2 n\left|q_{f}\right| B}-s \mu_{5}\right)^{2}\right)^{3 / 2}}-\frac{1}{\left(M_{0_{f}}^{2}+p_{z}^{2}+2 n\left|q_{f}\right| B\right)^{3 / 2}}\right],
$$


and

$$
\mathbf{I}_{3, \log }=\frac{N_{c}\left|q_{f}\right| B}{(2 \pi)^{2}} \sum_{n=0}^{\infty} \sum_{s= \pm 1} \int d p_{z} \frac{M_{f}^{2}}{\left(M_{0_{f}}^{2}+p_{z}^{2}+2 n\left|q_{f}\right| B\right)^{3 / 2}} .
$$

It can be shown that the term $\mathbf{I}_{3, \text { finite }}$ is finite. On the other hand, the term $\mathbf{I}_{3, \log }$ is not convergent at large momenta. Using Eqs. (D2), (D7), and (D10), Eq. (D1) can be rearranged in the following way:

$$
\begin{aligned}
\frac{\partial\left\langle\bar{\psi}_{f} \boldsymbol{\psi}_{f}\right\rangle_{\mathrm{vac}, \mathrm{B} \neq 0}^{\mu_{5} \neq 0}}{\partial M_{f}}= & \mathbf{I}_{1, \text { quad }}-\frac{M_{0_{f}}^{2}-M_{f}^{2}+2 \mu_{5}^{2}}{2} \mathbf{I}_{1, \log }+\mathbf{I}_{1, \text { finite } 1}+\mathbf{I}_{1, \text { finite } 2}+\mathbf{I}_{2, \text { finite }}+\mathbf{I}_{3, \text { finite }}+\mathbf{I}_{4}+\mathbf{I}_{2, \log }+\mathbf{I}_{3, \log } \\
= & -\frac{M_{0_{f}}^{2}-M_{f}^{2}+2 \mu_{5}^{2}}{2} \mathbf{I}_{1, \log }+\mathbf{I}_{1, \text { finite } 1}+\mathbf{I}_{1, \text { finite } 2}+\mathbf{I}_{2, \text { finite }}+\mathbf{I}_{3, \text { finite }} \\
& +\left(\mathbf{I}_{4}+\frac{N_{c}\left|q_{f}\right| B}{(2 \pi)^{2}} \int d p_{z} \frac{M_{f}^{2}}{\left(M_{0_{f}}^{2}+p_{z}^{2}\right)^{3 / 2}}\right)+\left(\mathbf{I}_{1, \text { quad }}+\mathbf{I}_{2, \log }\right) \\
& +\left(\mathbf{I}_{3, \log }-\frac{N_{c}\left|q_{f}\right| B}{(2 \pi)^{2}} \int d p_{z} \frac{M_{f}^{2}}{\left(M_{0_{f}}^{2}+p_{z}^{2}\right)^{3 / 2}}\right) \\
= & -\frac{M_{0_{f}}^{2}-M_{f}^{2}+2 \mu_{5}^{2}}{2} \mathbf{I}_{1, \log }+\mathbf{I}_{1, \text { finite } 1}+\mathbf{I}_{1, \text { finite } 2}+\mathbf{I}_{2, \text { finite }}+\mathbf{I}_{3, \text { finite }}+\mathbf{I}_{\text {finite }}+\mathbf{I}_{\text {quad }}+\mathbf{I}_{\log },
\end{aligned}
$$

where $\mathbf{I}_{\text {finite }}$ is

$$
\mathbf{I}_{\text {finite }}=\mathbf{I}_{4}+\frac{N_{c}\left|q_{f}\right| B}{(2 \pi)^{2}} \int d p_{z} \frac{M_{f}^{2}}{\left(p^{2}+M_{0_{f}}^{2}\right)^{3 / 2}},
$$

and

$$
\begin{gathered}
\mathbf{I}_{\text {quad }}=\mathbf{I}_{1, \text { quad }}+\mathbf{I}_{2, \log } \\
=-\frac{N_{c}\left|q_{f}\right| B}{2 \pi^{2}}\left[x_{0_{f}}\left(1-\ln x_{0_{f}}\right)+\ln \Gamma\left(x_{0_{f}}\right)+\frac{1}{2} \ln \left(\frac{x_{0_{f}}}{2 \pi}\right)\right]-\frac{2 N_{c}}{(2 \pi)^{3}} \int_{|\vec{p}| \leq \Lambda} d^{3} p \frac{1}{\sqrt{p^{2}+M_{0_{f}}^{2}}} . \\
\mathbf{I}_{\log }=\mathbf{I}_{3, \log }-\frac{N_{c}\left|q_{f}\right| B}{(2 \pi)^{2}} \int d p_{z} \frac{M_{f}^{2}}{\left(p^{2}+M_{0_{f}}^{2}\right)^{3 / 2}}, \\
=-\frac{N_{c} M_{f}^{2}}{2 \pi^{2}}\left[-\ln x_{0_{f}}+\frac{1}{2 x_{0_{f}}}+\frac{\Gamma^{\prime}\left(x_{0_{f}}\right)}{\Gamma\left(x_{0_{f}}\right)}\right]+\frac{2 N_{c}}{(2 \pi)^{3}} \int_{|\vec{p}| \leq \Lambda} d^{3} p \frac{M_{f}^{2}}{\left(p^{2}+M_{0_{f}}\right)^{3 / 2}},
\end{gathered}
$$

with

$$
\begin{aligned}
\mathbf{I}_{1, \log }= & \frac{N_{c}\left|q_{f}\right| B}{(2 \pi)^{2}} \sum_{s= \pm 1} \sum_{n=0}^{\infty} \int d p_{z} \frac{1}{\left(p_{z}^{2}+M_{0_{f}}^{2}+2 n\left|q_{f}\right| B\right)^{3 / 2}}=-\frac{N_{c}}{2 \pi^{2}}\left[-\ln x_{0_{f}}+\frac{\Gamma^{\prime}\left(x_{0_{f}}\right)}{\Gamma\left(x_{0_{f}}\right)}\right] \\
& +2 N_{c} \int_{|\vec{p}| \leq \Lambda} \frac{d^{3} p}{(2 \pi)^{3}} \frac{1}{\left(p^{2}+M_{0_{f}}^{2}\right)^{3 / 2}} .
\end{aligned}
$$


[1] S. Jeon and V. Koch, Phys. Rev. Lett. 83, 5435 (1999).

[2] M. Asakawa, U. W. Heinz, and B. Muller, Phys. Rev. Lett. 85, 2072 (2000).

[3] Rafelski and B. Muller, Phys. Rev. Lett. 48, 1066 (1982).

[4] P. Koch, B. Muller, and J. Rafelski, Phys. Rep. 142, 167 (1986).

[5] F. Karsch and E. Laermann, Phys. Rev. D 50, 6954 (1994).

[6] C. Bernard, T. Burch, C. DeTar, J. Osborn, S. Gottlieb, E. B. Gregory, D. Toussaint, U. M. Heller, and R. Sugar, Phys. Rev. D 71, 034504 (2005).

[7] M. Cheng et al., Phys. Rev. D 74, 054507 (2006).

[8] Y. Aoki, Z. Fodor, S. D. Katz, and K. K. Szabó, Phys. Lett. B 643, 46 (2006).

[9] M. Cheng et al., Phys. Rev. D 75, 034506 (2007).

[10] L. K. Wu, X. Q. Luo, and H. S. Chen, Phys. Rev. D 76, 034505 (2007).

[11] P. Zhuang, J. Hüfner, and S. P. Klevansky, Nucl. Phys. A576, 525 (1994).

[12] C. Sasaki, B. Friman, and K. Redlich, Phys. Rev. D 75, 074013 (2007).

[13] A. Smilga and J. J. M. Verbaarschot, Phys. Rev. D 54, 1087 (1996).

[14] D. Blaschke, A. Holl, C. D. Roberts, and S. Schmidt, Phys. Rev. C 58, 1758 (1998).

[15] P. Chakraborty, M. G. Mustafa, and M. H. Thoma, Phys. Rev. D 67, 114004 (2003).

[16] D. E. Kharzeev, L. D. McLerran, and H. J. Warringa, Nucl. Phys. A803, 227 (2008).

[17] V. Skokov, A. Yu. Illarionov, and V. Toneev, Int. J. Mod. Phys. A 24, 5925 (2009).

[18] V. Voronyuk, V. D. Toneev, W. Cassing, E. L. Bratkovskaya, V. P. Konchakovski, and S. A. Voloshin, Phys. Rev. C 83, 054911 (2011).

[19] W.-T. Deng and X.-G. Huang, Phys. Rev. C 85, 044907 (2012).

[20] J. Bloczynski, X.-G. Huang, X. Zhang, and J. Liao, Phys. Lett. B 718, 1529 (2013).

[21] L. McLerran and V. Skokov, Nucl. Phys. A929, 184 (2014).

[22] U. Gursoy, D. Kharzeev, and K. Rajagopal, Phys. Rev. C 89, 054905 (2014).

[23] V. Roy and S. Pu, Phys. Rev. C 92, 064902 (2015).

[24] K. Tuchin, Phys. Rev. C 91, 064902 (2015).

[25] I. A. Shovkovy, Lect. Notes Phys. 871, 13 (2013).

[26] V. Gusynin, V. Miransky, and I. Shovkovy, Phys. Rev. Lett. 73, 3499 (1994).

[27] V. Gusynin, V. Miransky, and I. Shovkovy, Phys. Lett. B 349, 477 (1995).

[28] V. Gusynin, V. Miransky, and I. Shovkovy, Phys. Rev. D 52, 4747 (1995).

[29] V. Gusynin, V. Miransky, and I. Shovkovy, Phys. Rev. D 52, 4718 (1995).

[30] A. Y. Babansky, E. Gorbar, and G. Shchepanyuk, Phys. Lett. B 419, 272 (1998).

[31] K. Klimenko, arXiv:hep-ph/9809218.

[32] D. Ebert, K. Klimenko, M. Vdovichenko, and A. Vshivtsev, Phys. Rev. D 61, 025005 (1999).

[33] M. Vdovichenko, A. Vshivtsev, and K. Klimenko, Phys. At. Nucl. 63, 470 (2000).
[34] V. C. Zhukovsky, K. Klimenko, and V. Khudyakov, Theor. Math. Phys. 124, 1132 (2000).

[35] T. Inagaki, D. Kimura, and D. T. Murata, Prog. Theor. Phys. 111, 371 (2004).

[36] T. Inagaki, D. Kimura, and D. T. Murata, Prog. Theor. Phys. Suppl. 153, 321 (2004).

[37] S. Ghosh, S. Mandal, and S. Chakrabarty, Phys. Rev. C 75, 015805 (2007).

[38] A. Osipov, B. Hiller, A. Blin, and J. da Providencia, Phys. Lett. B 650, 262 (2007).

[39] A. Osipov, B. Hiller, A. Blin, and J. da Providencia, SIGMA 4, 024 (2008).

[40] K. Klimenko and V. Zhukovsky, Phys. Lett. B 665, 352 (2008).

[41] D. Menezes, M. Benghi Pinto, S. Avancini, A. Perez Martinez, and C. Providencia, Phys. Rev. C 79, 035807 (2009).

[42] D. Menezes, M. Benghi Pinto, S. Avancini, and C. Providencia, Phys. Rev. C 80, 065805 (2009).

[43] S. Fayazbakhsh and N. Sadooghi, Phys. Rev. D 83, 025026 (2011).

[44] B. Chatterjee, H. Mishra, and A. Mishra, Phys. Rev. D 84, 014016 (2011).

[45] S. S. Avancini, D. P. Menezes, M. B. Pinto, and C. Providencia, Phys. Rev. D 85, 091901 (2012).

[46] G. N. Ferrari, A. F. Garcia, and M. B. Pinto, Phys. Rev. D 86, 096005 (2012).

[47] V. Elias, D. McKeon, V. Miransky, and I. Shovkovy, Phys. Rev. D 54, 7884 (1996).

[48] J. O. Andersen and R. Khan, Phys. Rev. D 85, 065026 (2012).

[49] J. O. Andersen and A. Tranberg, J. High Energy Phys. 08 (2012) 002.

[50] T. D. Cohen, D. A. McGady, and E. S. Werbos, Phys. Rev. C 76, 055201 (2007).

[51] T. D. Cohen, D. A. McGady, and E. S. Werbos, Phys. Rev. C 80, 015203 (2009).

[52] D. E. Kharzeev, Prog. Part. Nucl. Phys. 75, 133 (2014).

[53] M. Ruggieri, Phys. Rev. D 84, 014011 (2011).

[54] K. Fukushima, M. Ruggieri, and R. Gatto, Phys. Rev. D 81, 114031 (2010).

[55] J. Chao, P. Chu, and M. Huang, Phys. Rev. D 88, 054009 (2013).

[56] L. Yu, H. Liu, and M. Huang, Phys. Rev. D 90, 074009 (2014).

[57] L. Yu, H. Liu, and M. Huang, Phys. Rev. D 94, 014026 (2016).

[58] Z.-F. Cui, I. C. Cloët, Y. Lu, C. D. Roberts, S. M. Schmidt, S.-S. Xu, and H.-S. Zong, Phys. Rev. D 94, 071503 (2016).

[59] R. L. S. Farias, D. C. Duarte, G. Krein, and R. O. Ramos, Phys. Rev. D 94, 074011 (2016).

[60] M. N. Chernodub and A. S. Nedelin, Phys. Rev. D 83, 105008 (2011).

[61] V. V. Braguta, E.-M. Ilgenfritz, A. Y. Kotov, B. Petersson, and S. A. Skinderev, Phys. Rev. D 93, 034509 (2016).

[62] V. V. Braguta, V. A. Goy, E. M. Ilgenfritz, A. Y. Kotov, A. V. Molochkov, M. Müller-Preussker, and B. Petersson, J. High Energy Phys. 06 (2015) 094. 
[63] X. Sheng, D. H. Rischke, D. Vasak, and Q. Wang, Eur. Phys. J. A 54, 21 (2018).

[64] X.-L. Sheng, R.-H. Fang, Q. Wang, and D. H. Rischke, Phys. Rev. D 99, 056004 (2019).

[65] H.-Th. Elze, M. Gyulassy, and D. Vasak, Nucl. Phys B 276, 706 (1986).

[66] H.-Th. Elze, M. Gyulassy, and D. Vasak, Phys. Lett. B 177, 402 (1986).

[67] U. Heinz, Phys. Rev. Lett. 51, 351 (1983); Ann. Phys. (N.Y.) 161, 48 (1985).

[68] E. Wigner, Phys. Rev. 40, 749 (1932).

[69] S. R. De Groot. W. A. Van Leeuwen, and Ch. G. Van Weert, Relativistic Kinetic Theory (North-Holland, Amsterdam, 1980).

[70] P. Carruthers and F. Zachariasen, Rev. Mod. Phys. 55, 245 (1983).

[71] I. Bialynicki-Birula, Acta Phys. Austriaca. Suppl. 18, 111 (1977).

[72] N. Weickgenannt, X. Sheng, E. Speranza, Q. Wang, and D. H. Rischke, Phys. Rev. D 100, 056018 (2019).

[73] N. Armesto, F. Dominguez, A. Kovner, and M. Lublinsky, J. High Energy Phys. 05 (2019) 025.

[74] S. Mao and D. H. Rischke, Phys. Lett. B 792, 149 (2019).

[75] X. Sheng, R. Fang, Q. Wang, and D. H. Rischke, Phys. Rev. D 99, 056004 (2019).

[76] J. Gao, J. Pang, and Q. Wang, Phys. Rev. D 100, 016008 (2019).

[77] J. Gao, Z. Liang, Q. Wang, and X. Wang, Phys. Rev. D 98, 036019 (2018).

[78] G. Prokhorov and O. Teryaev, Phys. Rev. D 97, 076013 (2018).

[79] E. V. Gorbar, V. A. Miransky, I. A. Shovkovy, and P. O. Sukhachov, J. High Energy Phys. 08 (2017) 103.

[80] J. Gao, S. Pu, and Q. Wang, Phys. Rev. D 96, 016002 (2017).

[81] Y. Wu, D. Hou, and H. Ren, Phys. Rev. D 96, 096015 (2017).

[82] Y. Hidaka, S. Pu, and D.-L. Yang, Phys. Rev. D 95, 091901 (2017).

[83] K. Hattori, Y. Hidaka, and D.-L. Yang, arXiv:1903.01653.

[84] T. Hatsuda and T. Kunihiro, Phys. Rev. Lett. 55, 158 (1985).

[85] W. Florkowski and B. L. Friman, Acta Phys. Pol. B 25, 49 (1994).

[86] J. Hufner, S. P. Klevansky, P. Zhuang, and H. Voss, Ann. Phys. (N.Y.) 234, 225 (1994).

[87] S. P. Klevansky, Rev. Mod. Phys. 64, 649 (1992).

[88] M. K. Volkov, Phys. Part. Nucl. 24, 35 (1993).

[89] T. Hatsuda and T. Kunihiro, Phys. Rep. 247, 221 (1994).
[90] W. Florkowski, J. Huefner, S. P. Klevansky, and L. Neise, Ann. Phys. (N.Y.) 245, 445 (1996).

[91] W. M. Zhang and L. Wilets, Phys. Rev. C 45, 1900 (1992).

[92] A. Abada and J. Aichelin, Phys. Rev. Lett. 74, 3130 (1995).

[93] W. Florkowski, Phys. Rev. C 50, 3069 (1994).

[94] R. Gatto and M. Ruggieri, Phys. Rev. D 85, 054013 (2012).

[95] M. Ruggieri and G. X. Peng, J. Phys. G 43, 125101 (2016).

[96] S. S. Xu, Z. F. Cui, B. Wang, Y. M. Shi, Y. C. Yang, and H. S. Zong, Phys. Rev. D 91, 056003 (2015).

[97] B. Wang, Y. L. Wang, Z. F. Cui, and H. S. Zong, Phys. Rev. D 91, 034017 (2015).

[98] R. L. S. Farias, G. Dallabona, G. Krein, and O. A. Battistel, Phys. Rev. C 73, 018201 (2006).

[99] S. S. Avancini, A. Bandyopadhyay, D. C. Duarte, and R. L. S. Farias, arXiv:1907.09880.

[100] D. Vasak, M. Gyulassy, and H. T. Elze, Ann. Phys. (N.Y.) 173, 462 (1987).

[101] R. Fang, L. Pang, Q. Wang, and X. Wang, Phys. Rev. C 94, 024904 (2016).

[102] M. Buballa, Phys. Rep. 407, 205 (2005); M. Frank, M. Buballa, and M. Oertel, Phys. Lett. B 562, 221 (2003).

[103] V. Dmitrasinovic, Phys. Rev. C 53, 1383 (1996).

[104] L. Yang and X.-J. Wen, Int. J. Mod. Phys. A 33, 1850123 (2018).

[105] W. V. Liu and F. Wilczek, Phys. Rev. Lett. 90, 047002 (2003); E. Gubankova, W. V. Liu, and F. Wilczek, Phys. Rev. Lett. 91, 032001 (2003).

[106] J. Berges and K. Rajagopal, Nucl. Phys. B538, 215 (1999).

[107] J. Noornah and I. Shovkovy, Phys. Rev. D 76, 105030 (2007).

[108] G. Endrodi, J. High Energy Phys. 04 (2013) 023.

[109] M. Ferreira, P. Costa, D. P. Menezes, C. Providncia, and N. N. Scoccola, Phys. Rev. D 89, 016002 (2014).

[110] A. Abhishek and H. Mishra, Phys. Rev. D 99, 054016 (2019).

[111] D. C. Duarte, R. L. S. Farias, and R. O. Ramos, Phys. Rev. D 99, 016005 (2019).

[112] D. Boer and J. K. Boomsma, Phys. Rev. D 78, 054027 (2008); J. K. Boomsma and D. Boer, Phys. Rev. D 80, 034019 (2009).

[113] R. L. S. Farias, V. S. Timoteo, S. S. Avancini, M. B. Pinto, and G. Krein, Eur. Phys. J. A 53, 101 (2017).

[114] I. S. Gradshteyn and I. M. Ryzhik, Table of Integrals, Series and Products, edited by A. Jeffrey and D. Zwillinger (Elsevier Acadmic Press Publication, San Diego, California, 2007).

[115] http://mathworld.wolfram.com/HurwitzZetaFunction .html. 